\title{
Universität Bielefeld/IMW
}

\author{
Working Papers
}

Institute of Mathematical Economics

\section{Arbeiten aus dem \\ Institut für Mathematische Wirtschaftsforschung}

Nr. 12

Carl Christian von Weizsäcker

Grenzen der traditionelien GIObalsteuerung

Oktober 1973

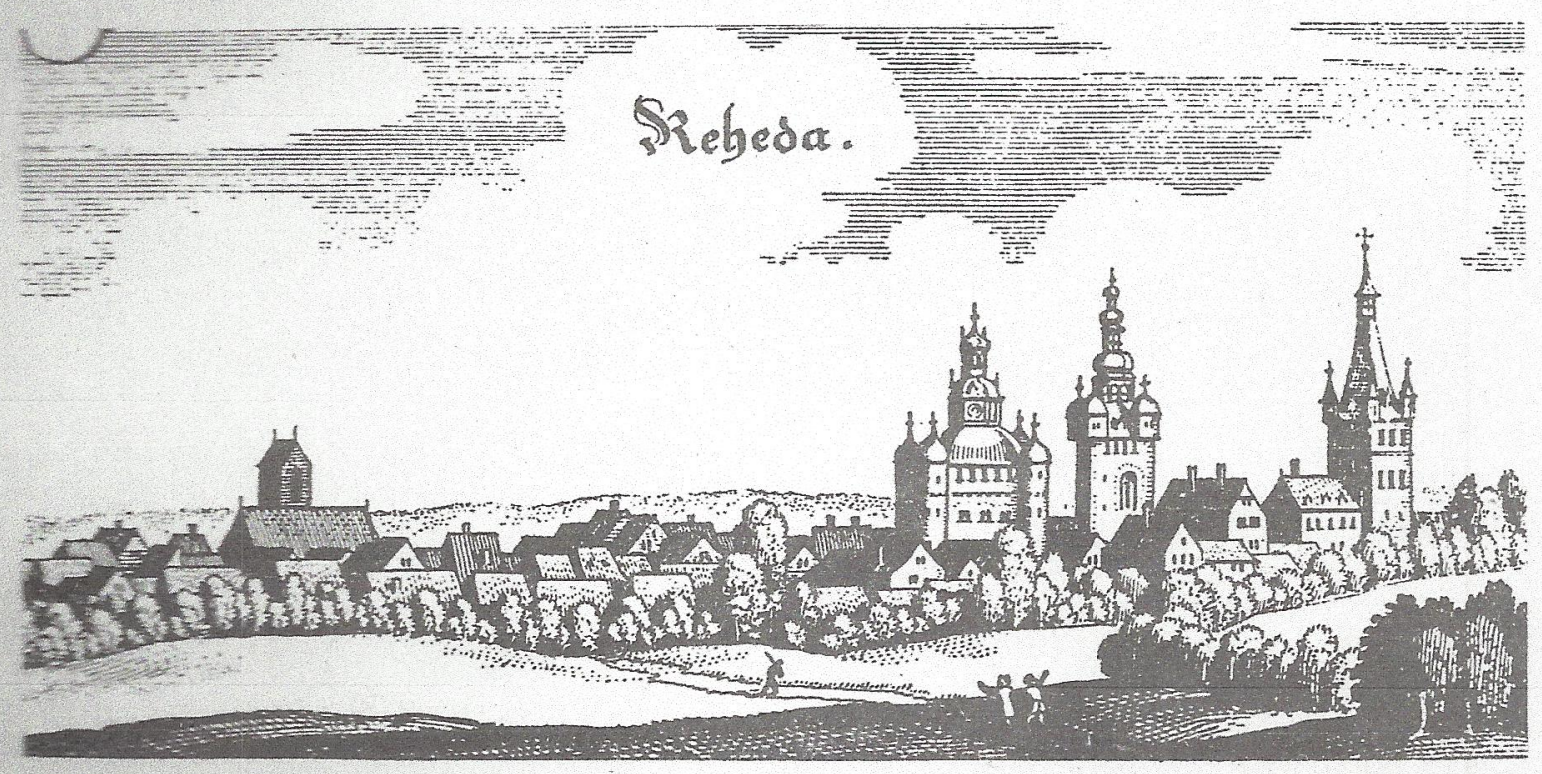

Institut für Mathematische Wirtschaftsforschung

an der

Universität Bielefeld

Adresse/Address:

Schloß Rheda

484 Rheda

Bundesrepublik Deutschland

Federal Republic of Germany 
C.C. von Weizsäcker

\section{Grenzen der traditionellen Globalsteuerung 1,2}

Die Enttäuschung ưber die Einsatzmo̊glichkeit und Effektivitåt der globalen Konjunktursteuerung ist das, was die wirtschaftspolitiker aller westilchen Länder heute miteinander verbindet. Die Probleme der stagflation sind dem konservativ regierten Grobbritannien und dem sozialdemokratisch geführten schweden gemein. Und die rasant steigenden preise engen gleichermaßen den Handlungssplelraum der liberaldemokratischen Regierung in Japan wie der sozialliberalen Koalition in der Bundesrepublik ein. In vielen Ländern steigt deshalb die Tendenz, die Wirtschaft dirigistisch zu lenken. Preiskontrolien der einen oder anderen form sind hierfir Beispiele. Wie an sich vorauszusehen war, sind diese Maßnahmen bisher ohne Erfolg.Der Dilet tantismus, mit dem sie durchgefuhrt werden, zeigt die Rat- und Planlosigkeit an, mit der mancherorts in westlichen Ländern heutzutage Wirtschaftspolitik getrieben wird. Wie aber fuiht der Weg weiter, wenn die Globalsteuerung nicht befriedigt und die Preiskontrollen eine Sackgasse sind? Hier kann man nicht antworten, wenn man nicht den richtigen Kern des Modells der Globalsteuerung erkennt und ihn aus seinem überzogenen Anspruch umfassender und zeitloser Gültigkeit herausschält. Diesen richtigen Kern zu leugnen, wäre dogmatisch und müBte politisch in die Irre fủhren. Um inn zu verstehen und für die Reform der Wirtschaftspolitik zu erhalten. müssen wir einige akademisch erscheinende Umwege in der Argumentation gehen. Der praktiker möge die folgenden theoretischen schnörkel verzeihen. Sie scheinen mir notwendig, wenn man darlegen will, weshalb die bisher versuchten oder vorgeschlagenen Alternativen zum traditionellen Konzept der Globalsteuerung unzureichend sind.

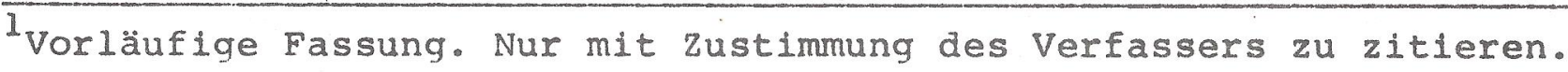

${ }^{2}$ Dieser Aufsatz richtet sich nicht nur an Fachkollegen in der Wissenschaft, sondern auch und gerade an die Praktiker der wirtschaftspolitik. Ich habe deshalb auf umständliche wissenschaftliche Präzisierung mittels eines ausgefeilten Begriffs- und ouellenapparats verzichtet. 


\section{Schwächen bisheriger Konjunkturpolitik}

\section{Monetaristen}

Die Monetaristen schlagen vor, daB die Konjunkturpolitik eines Landes vor allem darin bestehen soll, die verfugbare Geldmenge in der Volkswirtschaft mit einer konstanten prozentualen Rate zu vermehren. Dies Rezept wird mit zwei empirisch vergleichsweise gut fundierten Behauptungen begruindet:

1. es besteht ein relativ konstantes Zahlenverhältnis zwischen der Hoghe des nominellen Sozlalprodukts und der Höhe der Geldmenge einer Volkswirtschaft (dieses zahlenverhåltnis wird die Umlaufgeschwindigkeit des Geldes genannt).

2. Zentralbanken und Regierungen wirken mit ihren Eingriffen häufiger destabilisierend als stabilisierend. Die Monetaristen erwarten also, daB ihre Regel die Ronjunkturentwicklung besser stabilisieren werde als ein Regime des konjunkturpolitischen Interventionismus. Damit mo̊gen sie vielleicht recht haben, vor allem in Ländern mit kleinem Aussenhandelsanteil am Sozialprodukt. Wer sich auf eine seriöse wissenschaftliche Diskussion mit den Monetaristen einlässt, kann nicht umhin, zuzugeben, daB ihr wissenschaftlicher Fleiß ihnen eine eindrucksvolle empirische Basis fủr ihre Behauptungen verschafft hat. Wir werden auf die Schwierigkeiten einer monetaristischen Konjunkturpolitik zu sprechen kommen. Aber niemand kann an dem engen $\mathrm{Zu}-$ sammenhang zwischen Geldmenge und nominellen Sozlalprodukt vorbei. Eine politik der stabilisierung des Geldwerts, die diese monetaristische Grunderkenntnis außer Acht läBt, muB scheitern. Schon deshalb waren alle Experimente der Preiskontrolle in den letzten Jahren umsonst.

In folgender Tabelle werden die Geldmenge und das in jeweils drei Monaten erzeugte nominelle Sozialprodukt der Bundesrepublik einander gegenibergestellt. 


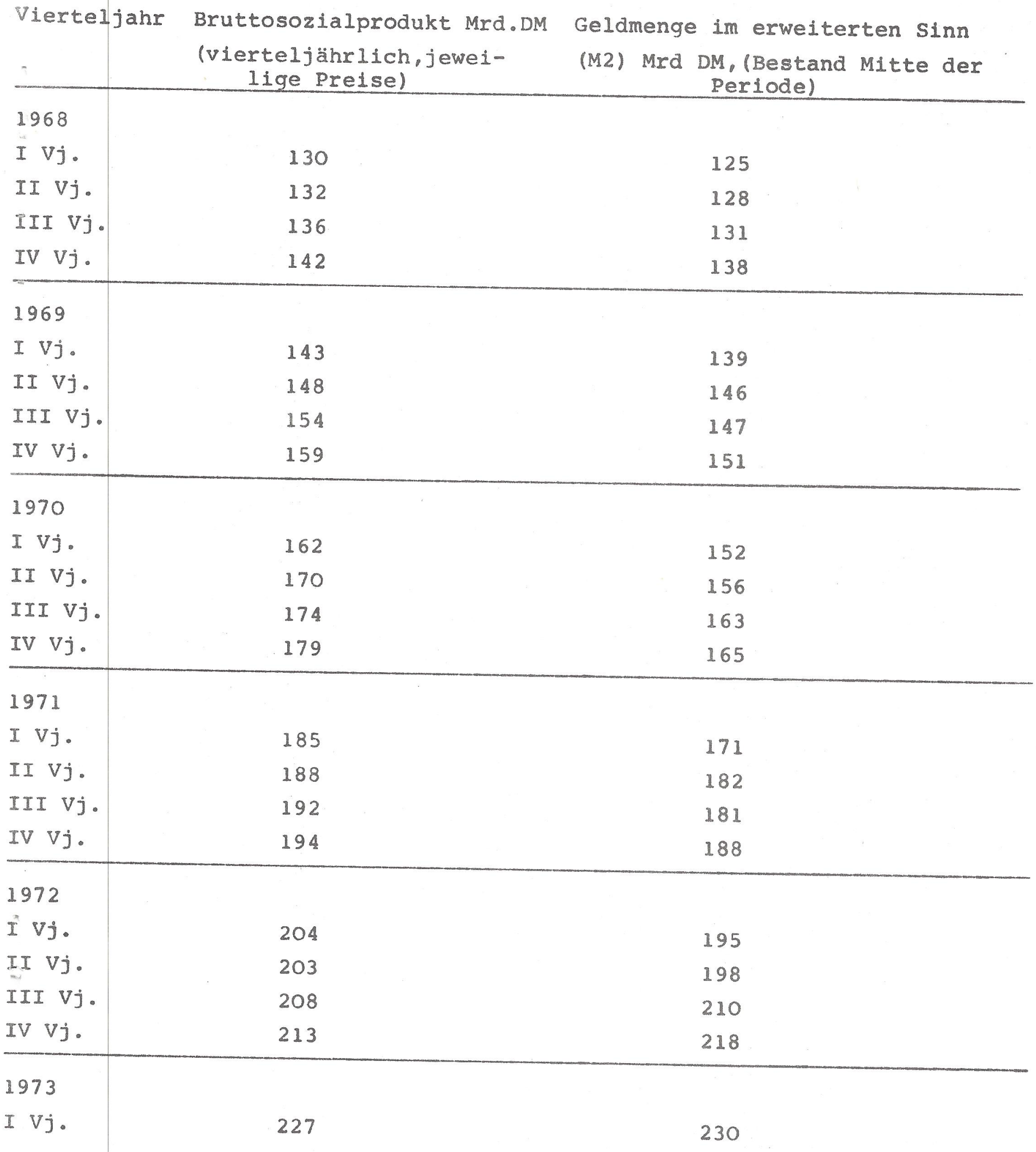

Quelle: Statistische Belhefte zu den Monatsberichten der Deutschen Bundesbank Reihe 4, August 1973, Tab. I und Tab. 24 
Der beschleunigte preisauftrieb der letzten Jahre, der sich im beschleunigten Wachstum des nominellen Bruttosozialprodukts widerspiegelt, läBt sich vom monetaristischen standpunkt aus voll durch die Erhöhung der Geldmenge erklären. ja er hätte, betrachtet man nur die Entwicklung der Geldmenge, sogar noch etwas stärker sein ko̊nnen.

Der Fehler des monetaristischen standpunkts ist nicht so sehr in den analytischen Details zu suchen als vielmehr in seiner politischen Naivität. Das hat das monetaristische Experiment in den Vereinigten staten in den Jahren 1969 und 1970 deutlich gezeigt. Die langen wirkungsverzögerungen konjunkturpolitischer Elngriffe mögen zusammen mit der unsicherheit über die zukunft ein gutes Argument der Monetaristen gegen den Versuch der Felnsteuerung (Friedman "fine tuning") der Konjunktur sein. Die gleichen Wirkungsverzögerungen gelten dann aber auch furr die selbsthellungskräte der Konjunktur, auf die sich die Monetaristen bel einer Politik des stetigen Geldmengenwachstums verlassen. Das lange Warten auf den Erfolg ist aber in einer parlamentarischen Demokratie unrealistisch. Der Bürger fuhrt die jeweils vorherschenden wirtschaftlichen Bedingungen auf die glelchzeitig durchgefihrten Maßnahmen der Reglerung zurůk, nicht auf solche, die schon lange zuríckliegen. Diese falsche Zurechnung auf Seiten des wählers macht elne strategie der bewubten konjunkturpolitischen Enthaltsamkeit politisch undurchfủhrbar: auch dann, wenn sie langfristig die beste Politik ist und die Eingriffe im Durchschnitt die Konjunkturschwankungen verstärken und die preisstabilität beeintrăchtigen. Was tut die Mutter des Kindes, dessen Arzt gegen sein bedrohliches Fieber nichts tun will, weil er es als notwendig firr die Hellung der Krankheit ansieht? Sie entläBt ihn und sucht einen anderen auf. Dabei spielt es keine Rolle, ob der erste Arzt objektiv recht hat oder nicht. 
2. Das Rezept flexibler Wechselkurse. Eine besondere Komplikation ergibt sich fur die monetaristische strateaie, wenn die aussenwirtschaftliche Verflechtung einer nationalen Volkswirtschaft nicht vernachlåssigt werden kann. Man kann die inländische Geldmenge natuirlich nur unter Rontrolle halten, wenn seitens der Zentralbank nicht die Verpflichtung besteht, fremde wăhrungen zu bestimmten Kursen gegen die eiqene wăhrung umzutauschen. Ein System der nationalen Geldmencenautonomie setzt deshalb ein system flexibler, oder wie man neuerdings sagt, "floatender" Wechselkurse voraus.

(Diese Aussage muss leicht modifiziert, bei långerfristiger Betrachtung aber nicht wesentlich geăndert werden, wenn man die heute vielfach benutzten Manipulationsmoiglichkeiten des Devisendiriqismus berücksichtiqt). So haben denn auch alle Monetaristen, zum Teil schon seit Jahrzehnten, ein system flexibler Wechselkurse befurwortet. Die genauere theoretische Analyse eines solchen systems (wie sie etwa in dem ausgezeichneten Buch Egon Sohmens "Wechselkurs und Wăhrungsordnung", Tưbingen 1973, in deutscher sprache vorliegt) zeigt, daß die meisten mehr technischen Einwände dagegen ihre Ursache mehr in der Denkfaulheit der Kritiker als in den Fehlern des systems selbst haben.

Der wesentliche Einwand gegen die Funktionsfähigkeit des reinen systems flexibler Kurse ist wiederum politischer Natur. Auch wenn seine Anhånger mit Recht argumentieren können, daß es im langfristigen Interesse der einzelnen staaten liege, sich an die spielregeln eines "sauberen" Floating zu halten, so werden die meisten staten dies dennoch nicht tun, sondern, dem Druck von Interessenoruppen oder der Notwendigkeit rascher Erfolge der Nachfragestimulierung sich beugend, offen oder heimlich auf den Devisenmärkten intervenieren. Wohl kann der Versuch gemacht werden, ein "schmutziges" Floatinq durch eine Rahmenvereinbarung zu verbieten. Ein "sauberes" Floating zu überprüfen ist nicht leichter als die Kontrolle einer Abri̊stungsvereinbarung. Wer etwa Devisen ankaufen will, um den Kurs der eigenen Wăhrung unter Druck zu halten, dies aber nach den spielregeln nicht darf, findet reichlich Möglichkeiten, die inländischen Geschåftsbanken so zu"beeinflussen" daB sie diese Aufgaben erledigen. Und selbst wenn diese Umgehung von Vereinbarungen nicht die 
Reqel sein sollte, so schürt jede ungewöhnliche Bewegung auf den Devisenmårkten den Argwohn, daß man heimlich ubervorteilt werde. Dies Klima des Argwohns ist langfristig einzig fư die Verstärkung protektionistischer Tendenzen zuträglich. Natůrlich impliziert andererselts auch der Versuch der stabl11slerung von Rursen abwertungsreifer währungen in einem system fixierter wechselkurse protektionistische Tendenzen.

Die Absicht dieser Kritik am reinen System flexibler Wechselkurse ist nicht, fưr ein system starrer kurse zu plädieren. Ein gro̊sseres MaB der Wechselkursflexibilitåt ist angesichts der schwäche supranationaler Institutionen politisch und ökonomisch notwendig und wird uns ja zur Zeit auch praktisch vorexerziert. Aber man sollte sich davor hiten, zu meinen, die größere Wechselkursflexibilität sei ein Schritt in Richtung auf einen von politischen Manipulationen weniger beeinflußten Welthandel. Man mag das bedauern, aber es scheint festzustehen: ein totaler welthandelsliberalismus ohne dirigistische, mexkantilistische, diskriminierende Elemente ist politisch genau so unrealistisch wie die monetaristischen Rezepte der konjunkturpolitischen Enthaltsamkeit. So ist es auch unrealistisch anzunehmen, in einem system der Wechselkursflexibilitat brauche die Zentralbank sich nicht mehr um den wechselkurs ihrer währung zu kuimmern. Gewiss hat sie mehr Handlungsfreiheit als in einem system fixierter wechselkurse, aber doch nicht so viel, daß sie in ihren Handlungen die Devisenmärkte ganz auf sich beruhen lassen könnte. Daher ist wohl auch mit einem Abbau des Devisendirigismus nicht zu rechnen. Dies gilt vor allem fuir Westeuropa, wo der Versuch unternommen wird, sich gegenseitig den Pelz zu waschen, ohne sich naß zu machen, d.h. eine währungsunion ohne Zentralisierung der Geldschöpfungskompetenzen zustandezubringen. 


\section{Fiskalpolitik und interventionistische Globalsteuerung}

Seit der Weltwirtschaftskrise und seit Keynes gehört es zu den Postulaten an die Wirtschaftspolitik, daß der Staatshaushalt auch als Instrument der Konjunktursteuerung verwendet werden solle. Durch Haushaltsdefizite soll der Konjunktur in Zeiten unzureichender Nachfrage aufgeholfen werden, durch Haushaltsüberschuisse soll in Boomperioden die Nachfrage gedämpft werden. So mochte man hoffen, die Gefahren der Depression - Arbeitslosigkeit und geringe Einkommen - und der Überkonjunktur - Inflation- zu bannen. Vollbeschäftigung bei stabilen Preisen schien eine realistische Vision. Die Bevölkerung konnte von allen grossen politischen parteien erwarten, daß sie sich nun für diese ziele einsetzen wirden. Der Erfolg im Erreichen dieser ziele und desjenigen eines angemessenen Wachstums des Lebensstandards wurde oft ausschlaggebend fir die Bestätiqung einer Regierungspartei an der Wahlurne. Die politischen Grenzen der Fiskalpolitik und der Geld-und Kreditpolitik zeigten sich jedoch im Verlauf der zeit immer mehr, und dies gerade, weil das technische Instrumentarium, das ihr zur Verfigung stand, so verbessert wurde, daß Mängel in der Globalsteuerung nicht mehr auf fehlende Instrumente zurückqeführt werden konnten. Besonders bei der Inflationsbekämpfung hat die Globalsteuerung nicht funktioniert. Die Grïnde hierfür sind leicht einzusehen.

Unterschiedliche Konjunkturlagen beruhen wesentlich darauf, daß Änderungen in der monetären Gesamtnachfrage sich nicht immer sofort in einer proportionalen f̈nderung des preisniveaus niederschlagen. Wäre dies nämlich der Fall, so wäre die reale Güternachfrage nicht durch monetäre Faktoren beeinflußbar. Es müsste dann immer der gleiche "normale" Konjunktur- und Beschäftigungszustand herrschen. Normalerweise reagiert das Preisniveau unmittelbar nicht sehr stark auf monetäre Nachfrageänderungen, so daß sich diese statt dessen auf das reale Sozialprodukt, die Beschăftigung und die Auslastung 
der Produktionsanlagen auswirken. Auf die Dauer bewirken dann zwar diese Menqeneffekte einer Nachfrageånderung ihrerseits Preiseffekte und Lohneffekte, die dann die ursprünglichen Mengeneffekte allmăhlich wleder beseitigen. Das Endresultat einer Veränderung der monetåren Nachfrage besteht in einer proportionalen Veränderung des Preisniveaus, wăhrend zum Schluss kaum noch ein Mengeneffekt vorhanden ist. Es ist aber wichtig, festzuhalten, daB bei Preis-und Lohnriqidität die Primăreffekte der staatlichen Nachfragebeeinflussunq Menqeneffekte sind, wăhrend die Preiseffekte oft Monate und Jahre auf sich warten lassen.

Damit ergibt sich aber eine vom politischen standpunkt aus entscheidende Asymmetrie zwischen expansiver und kontraktiver Globalsteuerung. Erwinscht ist der expansive Mengeneffekt, unerwinschte Begleiterscheinung ist der expansive Preiseffekt. Erwinscht ist umgekehrt der kontraktive Preiseffekt, der unerwinschte kontraktive Mengeneffekt muss dabei in Kauf genommen werden. Da die Menqeneffekte jeweils zeitlich vorangehen, kommt die expansive Politik unmittelbar in den Genuss ihrer Früchte. Ihre Kosten, der Preisauftrieb, fallen erst später an. Umgekehrt stellt sich vor das erwinschte Resultat der kontraktiven Politik - mehr Preisstabilität das unpopuläre "den-Gürtel-enger-schna llen." Es ist keiner Regierung, die auf das Vertrauen der wăhler angewiesen ist, zu verdenken, wenn sie angesichts dieser Asymmetrie mit der restriktiven Politik viel vorsichtiger hantiert als mit der expansiven. Die sucgestion einer symmetrie beider, die in einem formalen sinne natuirlich auch besteht, ist gewiss eine der politischen Naivitäten der herschenden akademischen politischen ökonomie.

Die politischen Schranken firr eine restriktive Politik der Globalsteuerung, insbesondere für eine restriktive Fiskalpolitik, haben die unmittelbare Folge, daß das ziel der 
Preisstabilitat unrealistischer wird, wahrend dadurch die Vollbeschäftigund nicht offenkundig beeinträchtigt wird: im Gegenteil, die genannte Asymmetrie in der Handhabung der Globalsteuerung scheint die Vollbeschäftigung im Vergleich zu einer symmetrischen Globalsteuerung zu beguinstigen. Im jeweils konkreten Fall, soweit man ihn isoliert betrachtet, ist dies auch richtig. Wenn eine Regierung trotz Preissteigerungen angesichts einer möglichen Rezession auf restriktive Maßnahmen verzichtet, so hilft sie ohne Zweifel furr die unmittelbare zukunft der vollbeschåftigung mehr, als wenn sie sich zu restriktiven Maßnahmen entscheidet. Besteht eine Prioritåt des zieles "Vollbeschäftigung" vor dem ziel "Preisstabilität" so kann diese als Begrůndung fur die Haltung der Reqierung dienen. Wird 5\% Inflation Im kommenden Jahr 5 영 Arbeitslosigkeit im kommenden Jahr vorgezogen, dann ist unter Umständen Vorsicht bei der Einsetzung restriktiver Maßnahmen auch dann geboten, wenn man schon mit $5 \%$ Inflation konfrontiert ist, aber noch Vollbeschäftigung genlesst. Anders sieht die situation aus, wenn man sich zu einer längerfristigen Perspektive entschliesst. Sachkundige Konjunkturpolitiker wissen, wie schwer es ist, von einem hohen Inflationsniveau, das sich seit längerem in die Volkswirtschaft eingenistet hat, wieder herunterzukommen. Arbeitgeber wie Arbeitnehmer, Kreditgeber wie Kreditnehmer, haben sich an diese Inflation gewöhnt und schllessen auf die Zukunft bezogene Tarifvertråge und Rreditvertråqe $a b$, die von der Erwartung ausgehen, daß die Inflation weitergeht. Wo wegen einer antizipierten Inflationsrate von $8 \%$ die Löhne um $12 \%$ erhöht wurden, käme es einer Katastrophe gleich, wenn die Absatzmårkte keine Preiserhöhungen zuliessen. Die Volkswirtschaft muss angesichts der auf Inflation gestimmten Erwartungen die Preissteigerungen der Vergangenheit in ungefähr gleicher Grössenordnung in der zukunft wiederholen, wenn sie eine schwere Rezession vermeiden will. 
Die kurzfristiqe für die Vollbeschäftigung nitzliche Asymmetrie in der Globalsteuerung hat längerfristiq den Nachteil, daß sich die Inflationserfahrung der Bevoilkerung auf ihre Zukunftsdispositionen auswirkt, sie inflatorisch konaitioniert und damit immer höhere Inflationsraten notwendig werden, wenn man die Vollbeschäftigung aufrecht erhalten will. Wenn bei einer Inflationsrate von 10\%, die in den Tarif-und Kreditverträgen auch schon für die Zukunft voll eskomptiert ist, eine Regierung die Konjunkturbremsen kräftig zieht und dadurch vor allem ein steigen der Arbeitslosenziffer, vielleicht dann auch ein Absinken der Inflation auf 8\% errelcht, dann hat man die beruhmte stagflation oder, wie es mancherorts in der den Rontinentaleuropåern eigenen Unhöflichkeit heißt: die "englische Krankheit". wer hilft dann dieser rachitischen Volkswirtschaft wieder auf die Beine? Und wie soll die Europaische wăhrungs- und Wirtschaftsunion vom Fleck kommen, wenn ihre Mitolieder einen Verein der staqflationäre darstellen?

Die These von der zunehmenden Inflationscewoohnuna erklärt sehr gut den Trend steidender Inflationsraten, die wir in allen westilchen Iändern beobachten. zu jedem geitpunkt exscheint es den Reqieruncen opportun, angesichts der dann herrschenden Inflationsrate und Inflationsqewöhnung der Bevolkerung einen Kurs einzuschlaqen, der im Interesse hoher Beschăftiqung $z u$ einer weiteren steigerung der Inflationsrate fihrt. zu jedem zeitpunkt erscheinen die kurz-und mittelfristiden Kosten einer schärferen Restriktionspolitik zu hoch, die doch notwendiq gare, um die langfristige Inflationsrate auch nur zu stabilisieren, erst recht, um sie zu reduzieren. Die Moraltheologen der Wirtschaftspolitik, die Professoren der Nationalökonomie, geisseln die sinden wider die stabilität, 
und nur selten finden sie sich bereit, den Regierungen, Parlamenten, Gewerkschaften, Banken, Exporteuren, Wählern Absolution zu erteilen. Vielleicht sollte ihnen qeraten werden, auch einmal vom Baum der politischen Erkenntnis zu essen. - Es ist für das weitere wichtig, sich mit dem Leser über die Erklärung der zunehmenden Inflationsraten zu verständigen. Es gibt zumindest eine andere Interpretation des von Konjunkturzyklus zu Konjunkturzyklus ansteigenden konjunkturunabhăngigen Inflationssockels. Sie wird z.B. vom DGB favorisiert, (vol. Rudolf Henschel, Wenn der Markt versagt, in "Die Zeit", 17.August 1973). Danach muss man die zunehmende konjunkturunabhängige Kormponente der Inflation erklären aus der zunehmenden Ausschaltung des Wettbewerbs auf vielen Markten, was seinerseits auf die Ronzentrationsprozesse und die starke stellung der multinationalen Konzerne auf diesen Mårkten zurickzufihren ist. Henschel etwa leitet aus dieser Analyse ab, daß entschiedene wettbewerbspolitische Maßnahmen und direkte staatliche Einqriffe zur Beschränkung der Marktmacht grosser Konzerne besonders vordringlich für die erfolgrelche Bekämpfung der Inflation seien. Einigen der wettbewerbspolitischen Folgerungen Henschels wirde ich durchaus zustimmen. Die Erklärung der gestiegenen Inflationsrate aus der zunehmenden Konzentration und Marktmacht erscheint aber zumindest unvollständig. Zum einen ist zu fragen, ob die Märkte heute eiqentlich so sehr viel mehr vermachtet sind als vor 20 Jahren. Man darf nicht vergessen, daß durch den gestiegenen internationalen Handel die Zahl der potenten ausländischen Ronkurrenten auf vielen Inlandsmärkten zugenommen hat, wodurch die Konzentration der inländischen Anbieter in ihrer Wirkung auf den Wettbewerb zum Teil oder ganz kompensiert worden ist. Auch hätte sich bei ausschliesslicher Gültigkeit der Henschelschen Erklärung die Verteilung des Volkseinkommens stärker zugunsten der Gewinne grosser Gesellschaften verschieben muissen als es im Trend der letzten 15 Jahre zu verzeichnen war. 
Ich kinnte mir aher vorstellen, daß eine stäkere oligopolstruktur auf den Märkten insofern indirekt zur inflatorischen Akzeleration beiqetragen hat, als dadurch die Riqidität der Preise gewachsen sein mad. Dadurch wiederum mag die zeitliche Verzögerung der preiseffekte von Globalsteuerungsmaßnahmen zugenommen haben: und dies wiederum mag AnlaB fior eine vermehrte präferenz von Reqierungen und Parlamenten firr expansive degenuber kontraktiven strategien gewesen sein, wodurch langfristiq entsprechend meiner oben angefủhrten Erklärung die Inflationsrate aestiegen ist.

4. Preiskontrollen. Die drei bisherigen Abschnitte iber die monetaristische strateqie, die flexiblen wechselkurse und die interventionistische Globalsteuerung setzte sich mit dem phänomen auseinander, daß sich die im Ansatz analytisch richtigen Vorschläge der akademischen ökonomen in der politischen Praxis nicht oder nicht sehr gut bewährt haben. Preiskontrollen sind von den professoren qanz überwiegend abgelehnt worden. Man hat dennoch mit ihnen in der Praxis experimentiert. Mit einer qewissen Genugtuund reqistriert man an den universitäten und Instituten, wie schlecht sie funktioniert haben. Noch im Herbst 1972 schien es so, als sel wenigstens das amerikanische Experiment nicht ganz umsonst qewesen. Inzwischen ist eingetreten, was Friedman und andere von Anfand an prognostiziert hatten: der Preiserhöhungsrilckstau hat sich auch in den USA mit voller wucht entladen, und dies zur ungelegensten zeit, nämlich gleichzeitiq mit der durch weltweite Verkna ppung hervorgerufenen exorbitanten Preissteigerung bei den Nahrungsmitteln. Naturlich muss zugegeben werden, daß ein politisches ziel mit den amerikanischen Preiskontrollen erreicht werden konnte. Zum zeitpunkt der wahlen im letzten Herbst herrschte eine vergleichsweise günstige Konjunktur bei einer vergleichsweise geringen Inflationsrate. Aber dieser Trick gelingt nur einmal. Ex rächt sich später, indem die Glaubwirdigkeit der Regierung auch in wirtschaftspolitischen Dingen beeinträchtigt wird, was ihre Handlungsfreiheit weiter eingrenzt. Mogen die Rezepte der National- 
ökonomen politisch naiv sein, so ist.es der dilletantische Opportunismus der Preiskontrollen, wie wir sie bisher beobachten konnten, letztlich auch. Fưr so dumm darf man den wähler auf Dauer nicht verkaufen wollen. Da im folgenden auch Vorschlåge zur Preiskontrolle gemacht werden, liegt mir daran, dem Leser diese meine Position zu den bisherigen Preiskontrollexperimenten deutlich zu machen.

II. Vorschläge zur Weiterentwicklung konjunkturpol1tischer Instrumente.

1. Ein Vorschlag zur besseren Anwendung von Preiskontrollen.

Wir hatten gesehen, daß die fir oligopolistische Mårkte charakteristische Preisrigiditat für die Bevorzugung expansiver Maßnahmen durch demokratisch gewählte Regierungen verantwortich gemacht werden kann. Die langfristige Folge dieser Bevorzugung ist eine im Trend steigende Inflationsrate, ohne daB auf Dauer die Vollbeschäftiqung besser garantiert wäre. Wenn die politische Aporie der Globalsteuerung ihre Wurzel in dieser prelsrigidität hat, dann hat die staatlich verordnete Preisrigiditat, die Preiskontrolle, natuirlich wenia beizusteuern zur Verbesserung der Konjunkturpolitik. Umgekehrt sollte man fragen: qibt es ein Verfahren, die Reaktion der Preise auf Maßnahmen der Globalsteuerung zu beschleunigen, die preise also flexibler, weniger rigide zu machen? Könnten Maßnahmen der Preiskontrolle hier eine Rolle spielen? Kann man die Prelskontrolle also in der entgegengesetzten Richtung einsetzen, nicht um staatlich verordnete Preisrigidität, sondern um statilch verordnete Preisflexibilität zu erlangen?

Maßnahmen zur Förderung des Wettbewerbs können unter konjunkturpolitischem Aspekt ja genau in diesem sinne verstanden werden. Sie haben natirlich auch eine wichtige 
ordnunaspolitische Funktion, die nicht Théma dieses Aufsatzes ist. Im konjunkturpolitischen Zusammenhang können sie letztlich nur die Funktion haben, die Preisflexibilitåt zu exhöhen. Es wäre aber eine Illusion zu meinen. daß wettbewerbspolitische Maßnahmen allein eine hinreichende preisflexibilitåt erreichen.

Preisrigidităt ist nur zum Tell Folge ibermåssiger, durch Wettbewerbspolitik zu bekămpfender Marktmacht. Einige andere Grủnde furr Preisrigiditåt sollen aufgezăhlt werden. Ein wichtiges Element der preisrigidität sind die staatlich sanktionierten oder gar staatlichen Monopole, deren Preise politisch beeinflußt sind. Dazu gehorren Bahn- und Posttarife,die Tarife des öffentlichen Nahverkehrs, Elektriz1tåts-, Gas-, wasserversorgung. Staatlich gelenkte Preise gibt es daruberhinaus bei der Kohle, bei Agrarprodukten, im Versicherungswesen, im Gesunaheitsbereich. All diese Preise zeichnen sich durch hohe Rigidität aus. Sie ändern sich vor allem, wenn die Kosten sich ändern, so wie dies fur fast alle Preise gilt, wenn man unter dem Regime der Prelskontrolle steht. Sodann gibt es zahlreiche wirtschaftsbereiche, in denen durchaus reger wettbewerb herrscht, wo aber dennoch die Preise nicht so håufig geåndert werden. GroBe Teile der Ronsumgůterindustrie, der Investitionsgiterindustrie und des Dienstleistungsgewerbes gehorren dazu. Längerfristig gestalten sich die Preise in diesen Bereichen durchaus nicht unăhnlich den preisen auf sog. vollkommenen Mărkten. Das heist, das Preisniveau in der Branche richtet sich auf längere sicht danach, welches die Kosten des "Grenzbetriebes" sind, wăhrend intramarginale Betriebe eine Effizienzrente zu erzielen vermögen. Kurzfristig hingegen verfügen die Firmen, da die Produkte ihrer Konkurrenten nicht genau die gleichen sind, über gewisse Spielråume der Preisfestsetzung. Da sie bezuiglich der Reaktionsweise der Kunden sich auch nicht sicher sein können, orlentieren sie ihre Preispolitik håufig an gewissen aus der Erfahrung gewonnenen Rentabilitätsnormen sowie an dem, was ihre Konkurrenten tun. Das Ergebnis ist, daB Preisveränderungen 
kurzfristiq vor allem kosteninduziert sind, wobei die den Kosten hinzugeschlagene Margen normalen Gewinns, wie auch die Kosten selbst, von Firma zu Firma verschieden sind und davon abhången, wieviel die Firmen jeweils in der Vergangenheit an Gewinnen haben erwirtschaften können. Sicherlich ist dieser weq der Preisfestsetzung in den genannten Wirtschaftsbereichen nicht der ausschliessliche, aber er ist doch sehr weit verbreitet. Man muss berioksichtigen, daß die Festlegung von Preisen eine Entscheidung bei grosser Unsicherheit darstellt. Die Sozialpsychologie weiss zu berichten, daB in solchen situationen das Gewisse $1 \mathrm{~m}$ Vergleich zum Ungewissen, wenn auch Relevanten, eine hervorrangende Bedeutung fưr die Entscheidungsfindung hat. Was aber relativ gewiss ist, das sind die Kosten und das sind die schon gefallenen Entscheidungen anderer Anbieter. So werden die eigenen Kosten und über die Imitation der anderen Rosten in der Branche zu der kurzfristig vielleicht wichtigsten LeitIinie für die Preisverănderungen. Das aber bedeutet, daß viele preise nur langsam auf Veränderungen der Nachfrage situation reagieren. Insbesondere gilt diese Beobachtung für konsumnahe Branchen. Eine Hauptkomponente der Kosten sind die Lobne. Vielfach werden also preise auf die veränderte Gesamtnachfrage erst dann reagleren. wenn diese Einfluss auf die Löhne gewonnen hat, etwa durch eine veränderte Beschåftigungslage. Dieser langwierige Prozess der indirekten Preisbeeinflussung über die Kostenbeeinflussung ist natiorich mit unter das Phänomen der preisrigiditåt zu rechnen, die eine Inflationsbekămpfung liber die Globalsteuerung kostspielig, langwierig, risikoreich und unpopulär macht. Preisrigidität ist hier nicht im absoluten sinne gemeint, sondern nur bezůglich Veränderungen in der Nachfrage. Preise, die bezůglich Nachfrageänderungen rigide sein ko̊nnen, können zur gleichen zeit z.B. bezioglich Veränderungen der preise von Konkurrenzprodukten sehr reagibel sein (vgl. die oben angesprochene Imitationstendenz bel Unternhmen, wie bei Menschen überhaupt). Wettbewerbspolitik wird gegenuber dieser 
Form der preisrigidität natürich wenig ausrichten. Denn sie beruht, wie gesagt, auf der situation der Unsicherheit, in der sich die meisten Unternehmen einer dynamischen wirtschaft befinden. Sie ist der Preis des Fortschritts und der ständigen Umgestaltung des qualitativen und quantitativen Güterangebots im Verlauf des wachstums von volkswirtschaftlicher produktivität und Lebensstandard der Bevo̊lkerung. Dort, wo das Motiv fur Wettbewerbsbeschrånkungen die Ausschaltung der unternehmerischen Marktunsicherheit ist, hat elne erfolgreiche Wettbewerbspolitik sogar ein Mehr an unternehmerischer Unsicherheit zur Folge und das mit Recht; denn vor allem das ståndige Knelppbad des Marktes und seiner Risiken läBt die Unternehmer mehr fur den wirtschaftlichen Fortschritt lelsten, als man es staatlich beamteten wirtschaftsbirrokraten auch noch zutrauen darf (sagte der Professor und Beamte auf Lebenszeit).

Unser Vorschlag zur staatich verordneten Preisreagibilitat sieht nun wie folgt aus. Nimmt man ein par staatlich nicht regulierte Agrarmarkte aus, so herrscht eine situation vor, in der ein gewiBes Maß an Preisrigiditåt zu beobachten ist. Fủr diesen uiberwiegenden Bereich der Volkswirtschaft ordnet die Regierung in jedem Vierteljahx eine durchschnittliche Preisänderungsrate (Preissenkung oder Preiserhöhung) an. Sie kann diese angeordnete durchschnittliche Preisånderungsrate aber nicht autonom bestimmen: vielmehr muß sie kraft gesetzlicher Verpflichtung diese Preisånderungsrate an die Veränderung der Geldmenge in der Volkswirtschaft wăhrend des vergangenen Vierteljahres anpassen. Die Preisånderungsrate wird nicht gleich dieser Geldmengenånderungsrate sein, da das reale wachstum des Sozialprodukts schon bel preisstabilität eine Erhöhung der Geldmenge erfordert. Aber die beiden GröBen sollen doch in einer festen,gemäB einer gesetzlich festgelegten Formel zu berechnenden Beziehung zueinander stehen: durchschnittliche Preisånderung (prozentual) = Geldmengenånderung (prozentual) im vergangenen Vierteljahr minus $x$, wobei $x$ sich nach dem durchschnittlichen vierteljähriichen zuwachs des realen sozlalprodukts in den vergangenen Jahren bemisst. Bis jetzt unterscheldet sich der 
Vorschlag kaum von einem staatlich verordneten Preisstopp, wenn auch das preisniveau an die Geldmenge gekettet wird. Damit das Preisniveau um z.B. I Prozent steigt, ist es aber natuirlich nicht notwendig, alle preise um genau 1 prozent zu steigern. Es kommt ja nur auf den durchschnittichen Anstieg an. Welche Preise im einzelnen um wieviel steigen oder fallen, das soll durch die unternehmen im wesentlichen autonom bestimmt werden. Wie aber kann man einen durchschnittlichen Preisanstieg verordnen, wenn man die unternehmerische Freiheit der Preisgestaltung im wesentIichen aufrechterhålt? Der staat vergibt zu Beginn des Vierteljahres Prelserhöhungslizenzen an die einzelnen Unternehmen. Eine Lizenzeinheit lautet auf 1 prozent Preiserhöhung fuir $\mathrm{z} . \mathrm{B}$. 1000 DM Wertschöpfung (= Umsatz + Vorratsverănderung abzüglich vorleistungen anderer Unternehmen abrüglich Abschreibungen). Elne Firma mit 2 Millionen DM viexteljährlicher Wertschöpfung erhålt also 2000 solche Lizenzkoupons. Diese Koupons sind transferierbar von Firma zu Firma. Zum Ende des Vierteljahrs mu aber jede Firma soviele Koupons in der Hand haben und dem stat abliefern als den Preiserhöhungen entspricht, die sie durchgefỉhrt hat. Hat $z$. B. obengenannte Firma ihre preise durchschnittlich um 2 Prozent erhöht, so muB sie bei einer Wertschöpfung von 2 Millionen DM 4000 Koupons abliefern. Sie hat sich also auf dem freien Markt fur Preiserhohungskoupons 2000 dazu kaufen mussen. Jemand anders, der ihr diese 2000 Koupons verkauft hat, hat seine Preise entsprechend weniger erhöhen können.

Technisch wird dieses system etwas anders funktionieren muissen: im Anhang sind einige Details seines Ablaufs skizziert. Der Grundgedanke ist aber sehr einfach: durch einen freien Markt der preisveränderungslizenzen kann eine durchschnittliche Preiserhöhungsrate durchgesetzt werden, ohne daB die für die Marktwirtschaft langfristig todliche staatliche Fixierung von allen einzelnen preisen notwendig wåre.

Natůrlich bleibt das problem bestehen, wie man ůberprůfen kann, ob die den staatlichen stelien gemeldeten preise den tatsåchlichen auch entsprechen. Im Prinzip ist das ein problem, das sich im Bereich der Besteuerung ebenfalls stellt. Dort gibt es etwa die Betriebsprüfung durch Finanzämter. Diese kann, wie man weiB, auch 
keine ideale Ubbereinstimmung zwischen tatsächlich gezahlter und elgentlich zu zahlender steuer herstellen. Man weiB, welche schwierigen Probleme der Bewertung von Aktiva, der Gewinnermittlung, etc. sich hier ergeben. Dennoch ist die zahl derer, die hieraus die Abschaffung der steuern als Forderung ablelten, sehr klein. Natủrlich engen diese schwierigkeiten der Besteuerungspraxis den Gesetzgeber in der Gestaltung von steuergesetzen erheblich ein. Auch die volkswirtschaftliche Besteuerungsquote ist aufgrund dieser praktischen probleme nicht beliebig vermehrbar. Entsprechend muB auf das problem der preisůberprủung bei vorschlăgen wie diesem auch Ruicksicht genommen werden. Nur kann das Uberprůfungsproblem nicht als Argument für eine pauschale Ablehnung ohne Detailsdiskussion herhalten.

Ich glaube nicht, daB sich sehr wesentliche Probleme der Überprüung gemeldeter preise ergeben. Zuerst sei festgehalten, daß sich die Preiserhöhungsbeschränkungen nur beziehen auf diejenigen Preiserhöhungen, die nicht einfach die Weitergabe von Preiserhöhungen der Vorlieferanten darstellen. Natürlich mu einem Einzelhåndler erlaubt sein, den Verkaufspreis seiner Ware um denselben Betrag (nicht um denselben Prozentsatz!) zu erhöhen, um den sein Einkaufspreis erhöht worden ist. Das aber bedeutet natůrlich, daB die uberprifung der Preise derjenigen waren, die nicht direkt an den Endverbraucher gehen, erleichtert wird durch die Kooperation der kåufer dieser Waren. Ist der Marktpreis fur die Koupons hinreichend hoch, so hat beispielsweise der Einzelhăndler ein Interesse daran, die Preiserhöhung seines Lieferanten auf eigene Preiserhöhungen anrechnen zu lassen, um Koupons zu sparen. Das geht aber natirilch nur, wenn die Preiserhöhung des Lieferanten deklariert worden ist. Ist ungekehrt der Preis für preiserhöhungskoupons niedrig, dann hat der Lieferant selbst ein Interesse, die preiserhöhung zu deklarieren, weil sie ihn wenig kostet, wăhrend ihre Deklaration zu einem späteren zeitpunkt teurer werden könnte. Auf der letzten Verkaufsstufe, der stufe der Konsummenten, ist wohl ein gewisses Maß an uberprüung von deklarierten preisen nötig. Aber auch hier kann man sich der kooperation der Råuferselte und der Verbraucherverbände versichern. Die staatliche Forderung des 
Verbraucherschutzes ist auch ohne den hier gemachten Vorschlag wichtig. Maßnahmen des Verbraucherschutzes werden aber ohne. Zweifel auch die Preisuiberwachung erleichtern. Im Anhang wird ferner im einzelnen dargelegt, weshalb die Marktpreise fuir die Preisånderungslizenzen nicht sehr hoch sein werden. Auch das erleichtert die Kontrolle, da ein Umgehen der Kontrolle nicht viel bringt. Im Anhang wird auch das Problem neuer Produkte, und damit im zusammenhang das Problem von Qualitătsveränderungen diskutiert.

Die Übereinstimung zwischen deklarierten und tatsächlichen Preisen scheint mix furr hochorganisierte volkswirtschaften kein wirkliches Problem zu sein. Die eigentlich interessante Frage ist, ob ein Vorschlag wie dieser die unternehmen veranlabt, effizienzmindernde Entscheidungen zu fällen. Bekanntlich ist ja längerfristig die Crux von konventionellen Preiskontrollen, daß das streben nach kostenvermindernden Rationalisierungen nachläßt oder gar abstirbt. Wenn nämlich Preiserhöhungen aufgrund gestiegener Kosten zugelassen werden, sonst aber verboten oder erschwert sind, dann mu sich das Interesse an Verbesserungen des Produktionsablaufs vermindern. Von diesen Effekten direkter staatlicher Preisintervention ist bei unserem Vorschlag nichts zu sehen. Eine Kostenüberprüfung findet nicht statt. Rostenerhöhungen als Legitimation filr Preiserhöhungen sind im wesentlichen nicht notwendig. Die einzige Ausnahme bildet die Anrechenbarkeit von Preiserhöhungen der Vorleistungen auf lizenzfreie Preiserhöhungen. Aber auch hier geht es ja nicht um die Möglichkeit, ein absolutes preiserhöhungsverbot zu überwinden. sondern nur darum, Kosten des Kaufs von Prelserhöhungslizenzen zu sparen. Da diese Kosten der Preiserhöhungslizenzen nicht exorbitant hoch sind, lohnt es sich furr das Unternehmen dennoch, vorprodukte zum zweck der Kostensenkung sparsam zu verwenden.

Gesetzt, daB unser Vorschlag in der praxis funktionsfähig ist, so könnte dies ein wesentlicher Beitrag fuir elne auf lange sicht günstige Konjunktur - und stabilisierungspolitik sein. Denn nun gilt nicht mehr, daß die preiseffekte einer restriktiven oder expansiven Geldpolitik erst lange nach den Mengeneffekten eintreten. Eine Vermehrung der Geldmenge führt spä- 
testens nach einem Quartal zu dex Erhöhung des Preisniveaus, die sich bisher erst nach Jahren voll gezeigt hat und eine verminderung des wachstums der Geldmenge schlägt sehr schnell auf eine verminderung des wachstums der Preise durch. Natưrlich bedeutet diese rasche wirkung auf die Preise, daß die Mengeneffekte der Geldpolitik schwächer werden. Damit ist aber eline wesentliche Voraussetzung fưr die Bevorzugung der expansiven vor der restriktiven Geld- und Kreditpolitik mit ihren langfristig stagflationären Auswirkungen beseitigt. Dies muB aber keine ungi̊nstigen Auswirkungen auf die Vollbeschäftigung haben, mit der wir uns im übrigen unten noch im einzelnen beschäftigen werden. Das ziel einer vergleichsweise groBen preisstabilität wird wieder realistisch. Man nehme $z . B$. an, daB das vorgeschlagene Instrumentarium der Bundesregierung und der Bundesbank im Sommer 1973 zur Verfůgung gestanden hätte. Der verschärfte stabilitätskurs håtte sofort Resultate zeitigen ko̊nnen. Es ist anzunehmen, daß dann die illegalen streiks unterblieben wären und daß die Tarifverhandlungen des kommenden Winters bereits in einem Klima der stabilität des Geldwerts hätten geführt werden können. Die leldigen langen wirkungsverzögerungen einer stabilitätspolitik existierten nicht mehr und machten damit die Wahrscheinlichkeit ihres rechtzeitigen Einsatzes grosser. Der Sommer 1973 in der Bundesrepublik ist ůbrigens auch durch eine situation gekennzeichnet gewesen, in der eine unmittelbare Gefahr fur die Vollbeschäftigung nicht bestand. So konnte man ohne zu großes Risiko den restriktiven kurs zumindest ein stück weit einschlagen. Ein schneller Erfolg, der dann auch die Tarifverhandlungen beeinflußt hätte, hätte es auch erlaubt, die Richtung so lange durchzuhalten, als vom stabilitatsziel benötigt ist, und sie andererseits rechtzeitig zu revidieren, um die vollbeschätigung nicht zu gefährden. Ob dies unter den heutigen Bedingungen möglich ist, bleibt abzuwarten.

Der hier gemachte Vorschlag ist natürlich nicht mit der pforte zum konjunkturpolitischen schlaraffenland zu verwechseln. Befindet sich z.B. ein Land schon in der situation der stagflation, so bleibt ihm auch mit dem hier entwickelten Instrumentarium die peinliche Alternative zwischen Arbeitslosigkeit oder noch mehr Inflation nicht erspart. Wenn die stagflation erst einmal da ist. 
kann nur eine längerfristige und komplizierte strategle allmåhlich aus der Misere heraushelfen. Diese strategie kann hier nicht diskutiert werden und hångt zudem wesentlich von den konkreten politischen und wirtschaftlichen Umständen des Landes ab. Hier ist die Rede von Möglichkeiten, das Instrumentarium zur Vermeidung des zustands der Stagrlation zu verbessern.

Die probleme der Kindererziehung mỏgen als Analogie fuir die Philosophie unseres Vorschlags dienen. Die Politiker unter meinen Lesern mögen verzeihen, wenn ich sie hier mit Kindern, die erzogen werden müssen, vergleiche. Das Kind, das z.B. eine Fremdsprache sprechen und lesen oder ein Musikinstrument spielen lernen soll, muB sich über Jahre anstrengen und abmuihen. Es wirde oft bald in seinem Eifer erlahmen, wenn ihm kein anderer Lohn winkte, als irgendwann in der zukunft einmal die Sprache oder das Musikinstrument zu beherrschen. Es braucht unmittelbare Gratifikationen furr seine Muihe, und diese sind deshalb in jedem guten Erziehungsplan vorgesehen. Nichts ist in der Erziehung, wie in der Konjunkturpolitik, wichtiger als die zeitliche Nähe von Anstrengung und Erfolg. Kinder, wie politiker, wie Wähler, wie Tarifpartner sind auch nur Menschen.

\section{Indexklause In}

Immer wieder wird die Einführung von Indexklauseln bei Iöhnen und Zinsen gefordert, und immer wieder lehnen die verantwortlichen Träger der Wirtschaftspolitik die Indexklauseln ab. Beide seiten in dieser Debatte verfügen über gewichtige Argumente. Vieles sprach bisher dafir. daß bei den gegenwärtigen Formen der Konjunktur- und Stabilitätspolitik Indexklauseln zumindest bei Tariflöhnen destabilisierend wirken. Andererseits ist die zunehmende Tendenz der Unterhöhlung abgeschlossener Tarifverträge durch Teuerungszuschläge während der Tariflaufzeit ein Anzeichen dafur, daB in einem hinreichend inflatorischen Klima sich de-facto-Indexklauseln zu entwickeln beginnen, die als Ersatz für die nicht zugelassenen tariflich vereinbarten Indexklauseln fungiexen, mo̊glicherweise dieselbe destabilisierende wirkung wie letztere ausủben, aber zudem das ordnungspolitisch, verteilungspolitisch und konjunkturpolitisch wichtige Institut der von Gewerkschaften und Arbeitgebern vereinbarten 
Tarifvereinbaruncen entwerten. Wenn schon Indexklausein unvermeidbar werden, dann ist es besser, diese offiziell zu sanktionieren und tariffähig zu machen, als sie in einem politisch gefahrlichen wildwuchs uberhand nehmen zu lassen. Implizite oder offizlell sanktionierte explizite Indexklausein sind unausweichlich, sobald die unsicherheit über die kunftige Entwicklung des Preisniveaus ins allgemeine Bewustsein getreten ist, sobald also die Konjunkturpolitik ủber keine hinreichende Kontrolle des Preisniveaus mehr verfügt. Eine Marktwirtschaft benötigt das Institut mittel- und langfristig gulliger verträge, und je komplexer die Produktionstechnologle wird, desto wichtiger sind solche Verträge. Diese Vertrăge - zumindest soweit sie die kủnftige Bezahlung von künftigen Leistungen zum Inhalt haben - sind nur möglich, wenn künftige Löhne und Preise approximativ kalkullerbar sind, oder wenn Indexklauseln aas Preisniveaurisiko ausschalten. Die volkswirtschaftlichen verluste der Erschwernis solcher Verträge sind schwer meBbar: sie sind langfristig aber erheblich.

Die Vertreter der offiziellen Politik nehmen hier oft - gerade in der Bundesrepublik - einen etwas unlogischen Standpunkt ein. Einerseits lehnen sie Indexklauseln mit dem Argument ab, dies sei eine Kapitulation vor der Inflation. Andererseits entschuldigen sie die faktisch eingetretene Inflation mit der Weltinflation, die man nicht verhindern korne und die ihre unvermeidbare Finwirkung auf das heimische Preisniveau habe, was einer Kapitulation vor den Wirkungen der Weltinflation gleichkommt. Je mehr der einzelne den doppelten Boden dieser Argumentation durchschaut, desto weniger hat die offizielle politik eine Chance, die Indexklauseln zu verhindern.

Wann wirken Indexklauseln oder ihre Korrelate wie Teuerungszuschläge destabilisierend? Dles ist insbesondere dann der Fall, wenn die wirkungsverzögerungen statilcher Ronjunkturpolitik auf das preisniveau lang sind, und wenn die Preise ůberwiegend kosteninduziert und nicht nachfrageinduziert veråndert werden. Unter diesen Bedingungen führen Preisniveausteigerungen ůber Indexklauseln 
zu automatischen Kostensteigerungen, die ihrerseits zu weiteren Preisniveausteigerungen führen, ehe etwa eingeleltete konjunkturdåmpfende Maßnahmen eine wirkung auf die preise haben konnnen. Bis dann diese wirkung eintrifft, hat sich die situation derart verschlechtert und sind derart starke Mengeneffekte der Restriktionsmaßnahmen zu verzeichnen, daß die Kosten der Preisstabilisierung zu hoch erscheinen. Die Politiker sind entmutigt und lassen die Inflationsprobleme links liegen. Die Indexklauseln verstärken also die Probleme der Konjunkturpolitik, ale wir elingangs geschildert hatten.

Ganz anders sähe die situation aus, wenn das Preisniveau durch staatliche MaBnahmen rasch und unmittelbar beeinflusst werden könnte. Dann nåmlich helfen Indexklauseln dem stabilislerungsgeschäft. Setzt man z.B. die Existenz eines systems der preisänderungslizenzen voraus, wie es oben vorgeschlagen wurde, so kann man nicht nur einen raschen Effekt der Geldpolitik auf die Preise, sondern über die Indexklauseln auch auf die Rosten - Löhne und Zinsen - erzielen. Gerade wenn aber preisånderungen stark kosteninduziert sind, ist dies von Vorteil, weil somit das von der Zentralbank uiber die Geldpolitik administrierte preisniveau uiber die Indexklauseln auf die kosten und von da rasch auf die "natürlichen" Preise wirkt. Somit wird die Diskrepanz zwischen administriertem und natürlichem prelsniveau kleiner, und damit sinken die Preise für Preisänderungslizenzen. Das aber macht das Leben mit den preisänderungslizenzen leichter, deren ziel die gröBere Effektivität einer Politik stabilerer Preise ist.

Man kann das problem der Indexklauseln insbesondere bel Tarifverträgen und Kreditverträgen in diesem Zusammenhang auch noch von einer anderen seite her beleuchten. Es hängt offensichtich eng zusammen mit der Frage, wer eigentlich vorwiegend das konjunkturelle Risiko trägt. Natuirlich ist niemand unberührt vom konjunkturellen Geschehen, aber dieses trifft doch die einzelnen Gruppen unterschiedlich stark. Besonders stark sind dem konjunkturellen Risiko zum Beispiel diejenigen Arbeiter und Angestellten ausgesetzt, deren Beschäftigung von der Konjunkturlage abhängt. Andere 
Arbeitnehmer, deren Beschäftigung im Prinzip gesichert ist, mögen bei schlechter Konjunkturlage wegen wegfallender uberstunden, etc. EinkommenseinbuBen erleiden, sle gehören zur Mittelgruppe vom Gesichtspunkt des Konjunkturrisikos aus. Schließlich gibt es die große Gruppe derjenigen, an ihrer spitze das rasant wachsende Heer der Staatsdiener, die ỉberhaupt keine konjunkturellen Einbußen zu erleiden haben. Diese letztere Gruppe trägt bestenfalls ein inverses und daher eigentlich perverses Konjunkturrisiko: je schlechter die Konjunkturlage ist, desto mehr können sie für ihr festes Gehalt und ihre Sparbuchzinsen kaufen. Die Ronzentration des konjunkturelien Rislkos auf vergleichsweise kleine Teile der Bevo̊lkerung erschwert aber die stabilitåtspolitik. Bei gleichmäBiger Verteilung der Risiken einer stabilitätspolitik entfallt auf jeden einzelnen nux ein ertrăgliches MaB an möglicher materleller ElnbuBe, das leicht wiegt, wenn es gegen die Chancen eines stabilen oder leicht vorauskalkulierbaren Preisniveaus gewogen wird. Demgegenuiber ist eine rigorose stabilitätspolitik nicht durchsetzbar, wenn sie einen Tell der Bevölkerung schwer trifft. Sie ist dann auch, insbesondere angesichts der potentiellen Arbeitslosen, vom moralischen standpunkt aus nicht vertretbar. Was bedeuten nun IndexklauseIn in diesem Zusammenhang? Wir wollen als Gedankenexperiment elnmal annehmen, daB die Tarifverträge und die Rreditvertrăge eine Klausel enthalten, nach der die zu zahlenden Tariflohne und zinsen nicht um die Verănderungsrate des Preisindex, sondein eines Indes der Konjunkturlage korrigiert werden sollen.Dann wären solche Indexklauseln ein Mittel,um einen Teil der Chancen und Risiken der Konjunktur auf die Lohnempfanger und Zinsempfänger zu ủbertragen, deren Konjunkturrisiko klein ist. Eine solche Indexklausel wirde das Konjunkturrisiko besser streuen und damit stabilitåtspolitik besser mo̊glich machen. Beispielsweise wirden konjunkturdåmpfende Maßnahmen der stabilitätspolitik die Lohn- und zinskosten der Unternehmen senken, damit ihre Tendenz zu Preiserhöhungen rascher redumieren, gleichzeitlg aber auch ihre Tendenz, Arbeltskrafte zu entlassen, vermindern.

Natuirlich ist dieses Gedankenexperiment unrealistisch. Indexklauseln von der genannten Art sind nicht durchsetzbar, denn Arbeitnehmer und sparer sind nicht an einem abstrakten Konjunktur- 
index, sondern an den Preisen der Konsumgüter interessiert, die sie kaufen wollen. AuBerdem, da die konjunktur ja auch von anderen Faktoren als denen der staatlichen Konjunkturpolitik abhängt, ist das Argument des destabilisierenden Effekts auch dieser Indexklausel nicht von der Hand zu we1sen. Anders sieht es aus, wenn man Indexklauseln, bezogen auf die Ronsumgüterpreise, verknủpft mit dem Vorschlag eines Lizenzsystems für Preisånderungen. Dann ist das preisniveau lelcht und schnell durch staatliche MaBnahmen zu beeinflussen, so daB der destabilisierende Effekt der Indexklauseln entfält. Andererseits kann diese Indexklausel in diesem System das Risiko staatlicher stabilitätspolitik besser uiber die gesamte Bevolkerung verteilen. Damit ist kaum mehr gesagt, als was schon gesagt wurde: die Indexklauseln helfen, administriertes und natürliches preisniveau näher aneinander zu halten. Indessen macht dieser Exkurs über die Risikoumverteilung durch Indexklauseln vielleicht deutlich, daß unter den genannten Umständen Indexklauseln auch den Effekt einer entschlossenen stabilitätspolitik auf die Beschäftigung zu mildern vermag.

\section{Vollbeschäftigung}

Konjunkturpolitik ist als solche noch nicht einmal so alt. Vor fünfig oder mehr Jahren waren Konjunkturen ein Schicksal, dem nicht auszuweichen war. Erst die probleme der weltwirtschaftskrise, sodann das allgemeine Erstarken der Gewerkschaften und die Keynesche Revolution in der ökonomie haben die Ronjunkturpolitik modernen stils geschaffen. Die genannten historischen Wurzeln der Konjunkturpolitik machen deutlich, daß Konjunkturpolitik immer auch Vollbeschäftigungspolitik sein mu. Andererseits zeigt die Erfahrung mancher Länder der westlichen welt, daß, wie schon eben erlåutert, eine implizite oder explizite Vollbeschäftigungsgarantie durch eine Vernachlässigung anderer Ziele der Konjunkturpolitik auch nicht erreicht werden kann. Die Stagflation lehrt uns: eine Vollbeschäftigung auf Kosten der Preisstabilität ist eine Vollbeschäftigung auf Pump. 
Die wirtschaftliche Zukunft wird mit solchen Hypotheken belastet, daB eines Tages auch die Vollbeschäftigungsgarantie des states für den Arbeitnehmer ein wertloses Besitztell wird.

Ist also die Vollbeschäftigungsgarantie ein unerfủllbarer Wunschtraum der Konjunkturpolitiker? Dies glaube ich nicht. Man sollte Instrumente entwickeln können, um Vollbeschåftigung dort auf Dauex zu erhalten, wo man sie heute geniesst und dort mit der zeit wieder herzustellen, wo sie verloren wurde. Allerdings wird es nicht leicht sein, eine Debatte um solche Instrumente ohne Emotionen zu fuhren: denn das Problem der Vollbeschäftigung ist unlösbar mit dem Problem der Vertellung des Sozialprodukts verbunden. Bei Verteilungsproblemen drohen sich aber immer analytische Aussagen mit Vorurteilen untrennbar zu vermischen. Niemand kann von sich behaupten, hier ohne Vorurteil zu argumentieren. Die folgenden Vorschläge sind begründet durch analytische Aussagen, deren Vermengung mit seinen Vorurteilen der Verfasser selbst nicht geeignet ist, klarzulegen.

Die Tarifautonomie ist ordnungspolitischer Ausdruck der verteilungspolitischen Neutralität des Staates in der Auseinandersetzung zwischen Arbeitgebern und Arbeitnehmern. Sie ist zugleich eine Garantie dafur, daB der staat nicht bestimmte Gruppen von Arbeitnehmern gegenüber anderen begünstigt oder benachteiligt. Sie ist schließlich Gebot einer marktwirtschaftlichen orientierung der wirtschaftspolitik, indem sie es den unmittelbar betroffenen parteien des Arbeitsmarktes überläBt, in Kenntnis der jeweils herrschenden Marktbedingungen Höhe und struktur der Tariflöhne zu vereinbaren. Die staatliche Konjunkturpolitik kann aber auch ohne direkten Eingriff in die Tarifpolitik erheblichen indirekten EinfluB auf die Tarifgestaltung nehmen. Eine Regierung, die verkủndet, sie werde durch entsprechende Nachfragebeeinflussung die Vollbeschäftigung in jedem Fall aufrechterhalten, wirkt auf Tarifverhandlungen ganz anders als eine Regierung, die erklärt, sie werde durch entsprechende Nachfragesteuerung jedenfalls die preisstabilität durchsetzen.

Im Interesse einer stabilisierung des preisniveaus und zur Vermeidung stagflationärer Tendenzen ist von manchen ökonomen schon der Vorschlag gemacht worden, man solle die Tarifautonomie zwar beibehalten, man solle aber jeweils von allen Lohnerhöhungen mittels 
Besteuerung und Rückerstattung an die Arbeitgeber einen solchen prozentualen Abzug vornehmen, daß faktisch nur Lohnerhöhungen uibrig bleiben, die im volkswirtschaftlichen Durchschnitt dem Produktivitätsfortschritt entsprechen.Dieser Vorschlag geht von der Hypothese aus, daß Tar1fauseinandersetzungen die Lohnquote nicht wesentlich beeinflussen können, so dab es nur daraur ankomme, mittels der Tarifvereinbarungen die relativen Löhne, also die Lohnstruktur festzulegen. Wenn das so wàre, dann spräche in der Tat einiges fur einen solchen vorschlag.

Faktisch aber käme dieses schema einem wesentlichen Eingriff des Staates in die Tarifautonomie gleich. Wesentlicher Bestand. teil der Tarifauseinandersetzung ist ja der Rampf um die Aufteilung des produkts zwischen unternehmen und Arbeitnehmer, zwischen Gewinn und Lohn, zwischen Investition und Konsum. EIn nachträgliches staatliches Zurủckkorrigieren des Ergebnisses dieses Verteilungskampfes kann nicht als vereinbar mit dem Grundgedanken der Tarifautonomie angesehen werden.

Folgender Vorschlag versucht, diesen Eingriff in den Tarifkampf zwischen unternehmen und Arbeitnehmern zu vermeiden. Andererseits zlelt ex doch darauf ab, die Handlungsfrelhelt der Tarifpartner dort einzugrenzen, wo dies im Interesse der kurz- oder langfristigen sicherung der Vollbeschäftigung nötig erscheint. Im Interesse der kurzfristigen sicherung der Vollbeschäftigung werden der Arbeitgeberseite Auflagen gemacht: im Interesse der Vermeidung von Inflation, Stagflation und Arbeitslosigkeit auf langere sicht werden die Tariflöhne durch statiliche Intervention noch einmal nachkorrigiert, wenn auch in ganz anderer weise als bei dem oben diskutierten Vorschlag.

Zentrales Element des Vorschlags ist die "Beschäftigungskurve" des einzelnen Unternehmens. Jedes Unternehmen soll angeben, um wieviel es die zahl der durchschnittlich bei ihm Beschaftigten im năchsten Halbjahr zu veråndern wïnscht. Dabei muB es aber nicht nur eine einzelne Zahl angeben, sondern es mus diese Angabe fur unterschiedliche hypothetische Werte einer Beschätigtensteuer machen, die erhoben wird pro zusätzlich im nåchsten Halbjahr durch- 
schn1ttlich beschåftigter Person. Diese steuer kann mo̊glicherwelse auch negativ sein; dann ist sie elne Subvention. Graphisch dargestellt wirde eine solche von der Firma XY halbjăhrlich einzureichende Beschäftıgungskurve z.B. so aussehen

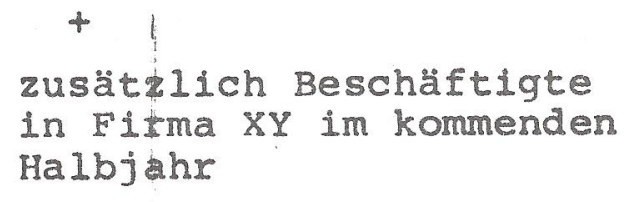

Beschåttigungssteuer pro zusătzlich Beschäftigten

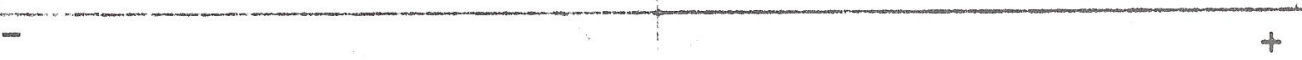

Je höher die Beschäftigungssteuer ist, desto kleiner wird die Firma die Beschäftigung halten wollen. Auch alle offentlichen Arbeltgeber mủssen eine solche Kurve für ihren Bereich angeben. Eine statliche stelle sammelt zu einem bestimmten Termin im Vorhalbjahr die Kurven der einzelnen Firmen und addiert sie zusammen zu einer volkswirtschaftlichen Beschäftigungskurve. Schon ehe die Firmen ihre einzelnen kurven eingereicht hatten, hat der staat eine volkswirtschaftliche Planziffer der zahl der im kommenden Jahr durchschnittlich Beschäftigten bekanntgegeben. Sie soll dem ziel 
dex Voll- aber nicht Úberbeschäftigung entsprechen. Nun liest der staat anhand der volkswirtschaftlichen Beschäftigungskurve ab, welche Beschäftigungssteuer erhoben werden müBte, damit die Zahl der Beschäftigten gemäß der Kurve der staatlichen planziffer der Beschäftigten entspricht. Diese den Ausgleich zwischen dem Plan des Staates und den Plänen der Arbeitgeber herbelfuhrende Steuerhöhe $\mathrm{x}$ (möglicherweise negativ) ist nun für das nåchste Halbjahr verbindich im folgenden sinn: jede einzelne Firma ůbernimmt die Verpflichtung, so viele Arbeitskräfte im Durchschnitt des năchsten Halbjahres zu beschäftigen, als ihrer Beschåftigungskurve bei einer Beschäftigungssteuer $x$ entspricht. Die Differenz zwischen dieser Beschäftiqungsziffer und der durchschnittlichen Beschäftigungsziffer des Vorhalbjahres wird nun mit der Beschäftigungssteuer $x$ belastet (oder es wird, sofern $x$ negativ ist, eine entsprechende subvention gezahit).

Wie schon im Vorschlag über die pxeisänderungslizenzen, ist auch hier vorgesehen, daß die Firmen ihre Beschäftigungsverpflichtung, bzw. -erlaubnis auf dem freien Markt anderen Arbeitgebern abtreten oder von diesen solche Beschäftigungslizenzen erwerben können. Dadurch können unnötige und unökonomische Einengungen der Handlungsfreiheit der einzelnen unternehmen vermieden werden, ohne daß das gesamtwirtschaftliche Beschäftigungsziel beeinträchtigt wird. Vollbeschåftigung kann keinesfalls heiBen, daß jedem Arbeitnehmer sein jeweiliger konkreter Arbeitsplatz garantiert wird. Technischer und sozialer Fortschritt, Änderungen der Nachfrage u.a.m. erfordern, daß immer neue Arbeitsplätze auch bei konstanter Erwerbsbevo̊lkerung geschaffen werden. Das aber ist nur möglich, wenn alte Arbeitsplätze verschwinden.

Ferner wird vorgeschlagen, daß eventuell anfallende Ertråge aus der Beschäftigungssteuer allen Arbeitnehmern in der Form eines proportionalen Lohnzuschlags ausgezahlt werden, also wie eine Lohnerhöhung wirken. Ist die steuer negativ, muissen also Beschäftigungssubventionen bezahlt werden, so werden diese entsprechend dadurch finanziert, daB von allen Lohnempeängern der gleiche prozentuale Abzug einbehalten wird. Wohlgemerkt be- 
deutet das im allgemeinen nicht, daB die Arbeiter eines Betriebes so viel abgezogen bekommen, wie dieser Betrieb an Subventionen erhält. Es findet durch diese Maßnahme ein Transfer zwischen verschiedenen Betrieben statt. Nur volkswirtschaftlich sollen sich Zahlungen aller Teilnehmer auf einer seite und Bezüge aller Teilnehmer auf der anderen seite gerade ausgleichen.

Hier ergibt sich der Zusammenhang des vorgeschlagenen schemas zur Tarifpolitik. Bei einer gegebenen stabilitätspolitik der Regierung und Zentralbank und bei gegebenen Erwartungen über d1. Konjunkturentwicklung mo̊gen gewisse Tarifabschlísse zu hoch sein, um Vollbeschäftigung erwarten zu lassen. Ohne dieses Schema läge nun der schwarze peter bei der Regierung, die das ziel dex Vollbeschäftigung proklamiert hat und deshalb gezwungen sein kann, ihren stabilitätskurs aufzugeben, um trotz der hohen Lohnsteigerungen die Vollbeschåftigung zu retten. Mit diesem schema jedoch ist die Vollbeschäftigung auch bei fortgesetztem Stabilitätskurs der Regierung kurzfristig garantiert. Es scheint allerdings gerechtfertigt, die Kosten der Vollbeschäftigungsgarantie von dort erstatten zu lassen, wo sie durch uberhöhte Lohnsteigerungen letztlich entstanden sind. Damit werden die Grenzen der Reallohnsteigerung bei Aufrechterhaltung der Vollbeschäftigung durch eine Korrektur der Nominallohne nach unten sichtbar gemacht, anstelle einer Rorrektur des Preisniveaus nach oben, die Folge einer Aufgabe des Stabll1tätskurses der Regierung wäre.

Kann aber ein solches Schema das Spannungsverhältnis zwischen den polen des magischen Dreiecks beseitigen? MuB nicht die verordnete Vollbeschäftigung in den Låndern, für die Vollbeschäftigung ein wirkliches Problem ist, zu so großen Lohnsteigerungen führen, daB die Tarifpartner sich an sehr hohe Lohnkorrekturen und Beschäftigungssubventionen gewöhnen? MuB die Antizipation dieser staatlichen Umvertellung von den Arbeitnehmern zu den Arbeitgebern nicht zu noch weiteren Lohnsteigerungen und noch höheren Beschäftigungssubventionen führen? Fuihrt dieses Karussell nicht binnen kurzem zu so exorbitant hohen Subventionen, daß dem Mißbrauch des systems nicht mehr 
gesteuert werden kann? Und bedeutet dies nicht seinen raschen Zusammenbruch mit all den katastrophalen Folgen eines radikalen Vertrauensschwunds?

Klar ist, daß das Schema nur funktionieren kann, wenn die Beschäftigungssteuer, bzw. vor allem die Beschäftigungssubvention sich in bescheidenen Grenzen hält. Es ist dies ein Grundgesetz "dirigistischer" Eingriffe. Ihre technische Funktionsfahigkeit hängt wesentlich davon $a b$, daB sich ein MiBbrauch oder eine Umgehung nicht zu sehr lohnt. Hohe zölle provozieren Schmuggel, hohe Steuern deren Hinterziehung, vom Verbot des Rauschgifthandels leben ganze Branchen der Unterwelt, bůrokratische Investitionskontrollen haben in vielen Ländern zu eintrăglichen Korruptionspfründen für Staatsbeamte und Minister geführt, Kapitalverkehrskontrollen verleiten zu Koffergeschäften und zur legalen Umgehung über Außenhandelsverflechtungen, Preiskontrollen können Schwarzmärkte entstehen lassen.

Daß sich das Schema der garantierten Vollbeschäftigung bei vergleichsweise niedrigen Steuer- und Subventionssätzen stabilisieren ließe, erwarte ich aus folgendem Grund. Wie schon gesagt, bedeutet eine globale Vollbeschäftigungsgarantie keine Garantie für jeden konkreten Arbeitsplatz. Ja sie hat nicht einmal zur Folge, daB man innerhalb einer Branche mit einer festen Zahl von Arbeitsplätzen rechnen kann. Auch regionale Verschiebungen der Beschäftigung sind mo̊glich. Dies aber bedeutet, daß Tarifverhandlungen in den einzelnen Branchen immer auch die branchenspezifische Beschäftiqungssituation berücksichtigen mússen. Insbesondere in den Branchen, in denen $z . B$. wegen guter gewerkschaftlicher Organisation schon vergleichswelse gute Löhne gezahlt werden, entsteht furr den einzelnen Arbeitnehmer das Risiko, daß ưberzogene Lohnsteigerungen ihm seinen Arbeitsplatz kosten, und er fir weniger Geld in elner anderen Branche arbeiten muß. So wird gerade in den Branchen, die tonangebend furr die Tarifabschlüsse im Land sind, das Gebot gesamtwirtschaftlicher Vernunft auch zum Gebot der partikulären Vorteile. Zugleich hat dies Schema der globalen Vollbeschåftigung damit einen egalitåren Effekt auf die branchenmäBige Lohnstruktur - und damit möglicherweise auch einen solidarisierenden Effekt in der Arbeit- 
nehmerschaft. Die globale Vollbeschäftigungsgarantie stårkt den Rỉcken der Arbeitnehmer in den Branchen, Betrieben und Lohngruppen, wo am schlechtesten bezahlt wird. Dies ist im übrigen auch vorteilhaft im sinne eines volkswirtschaftlich rationellen Einsatzes der Arbeitskräfte.

Können aber nicht die unternehmen durch eine verzerrte wiedergabe ihrer eigenen Beschåftigungsplåne die Beschåftigungssteuer kủnstlich senken, bzw. die Beschåftigungssubvention kủnstlich erhöhen? Entsteht ihnen dadurch nicht die Mo̊glichkeit, die Tarifvereimbarungen zu unterlaufen? Dies wăre der Fall, wenn die Arbeitgeber zusammengenommen ein monolithischer Block wären. Das aber ist nicht der Fall. Es ist bekannt, wie sehr bei entsprechender Konjunkturlage die unternehmen um Arbeitskräfte konkurrieren. Eine politik des gemeinsamen Verschiebens der Beschåftigungskurven zum zweck der Erhöhung von Beschäflgungssubventionen mu schon an der mangelnden Uberprůbarkeit scheitern.Denn die koordinierende Instanz der Arbeitgeber welB gar nicht, was denn die eigentiche Beschäftigungskurve der Unternehmen ist. So könnten die einzelnen Unternehmen ohne weiteres ihre richtige Beschäftigungskurve der staatlichen stelle angeben, ihrem Arbeitgeberkoordinationsgremium jedoch erzåhlen, es sei dies die gemäB Absprache verschobene Kurve. Im Gegensatz zu einem Kartellpreis oder elnem Kartellkontingent ist diese Beschäftigungskurve etwas viel zu firmensperifisches und unủberprüfbares als daß besagtes Arbeltgeberkartell gelingen konnte, zumal es alle Arbeitgeber der Volkswirtschaft, auch den stat eelbst, umfassen mủBte. Das beste und risikoloseste, was ein Unternehmen machen kann, ist, seine vom eigenen, isolierten Gewinnstandpunkt aus optimale Beschäftigungskurve anzugeben. Gibt das einzelne Unternehmen eine verschobene Kurve an, so hat dies ja nur einen prozentual kaum merkbaren Effekt auf die gesamtwirtschaftliche Beschåtigungskurve. Das gilt selbst fuir so grobe einzelne Arbeitgeber wie die Bundesbahn oder den SiemensKonzern. Der wesentliche Effekt der verschobenen Kurve wäre also nur, daß das Unternehmen eine andere Zahl von Beschäftigungslizenzen, bzw. -verpflichtungen erhält als es eigentlich winscht. 
Eines der großen Probleme der Tarifpolitik ist die Unsicherhelt über die künftige Konjunkturlage. An sich wären deshalb Indexklauseln, die Lohnrevisionen je nach Konjunkturlage vorsehen, nicht schlecht. Sie sind aber wohl nicht durchfuhrbar.uber die Beschäftigungslizenzen und das entsprechende Besteuerungs-, bzw. Subventionssystem wird etwas Ahnliches erreicht. Geht nach Abschluß von Tarifvertrăgen das konjunkturelle stimmungsbarometer nach oben, so wird sich dies beim nåchsten Termin zur Erstellung einer Beschäftigungskurve darin åußern, daß die Beschåftigungswinsche der Unternehmen steigen. Um die Beschäftgung zu stabilisieren, ist eine höhere Beschäftigungssteuer, bzw. eine geringere Beschäftigungssubvention als bisher nötig. Da der Ertrag der stever den Arbeitnehmern zufließt, bzw. die Subvention von ihnen aufgebracht wird, bedeutet dies automatisch eine Lohnerhöhung der Arbeitnehmer. Umgekehrt wirkt ein nicht vorausgesehener konjunkturabschwung. Unter solchen Bedingungen kann erwartet werden, daß die Tarifpartner in verninftiger weise die konjunkturelle Entwicklung in ihre Verhandlungen mit einbeziehen, was heute, beim gegenwärtigen system, immer schwerer wird. Zweimal in den letzten Jahren muBten die Gewerkschaften es erleben, daB tarifpolitische zurỉckhaltung, motiviert vor allem auch durch die Einschåtzung der konjunkturellen Lage, in dem dann folgenden, abex nicht vorausgesehenen Boom zu illegalen streiks fuhrte, die auch mit Lohnaufbesserungen honoriert wurden. Dies mu dazu fihren, daB auf die pauer konjunkturell adäquates Tarifgebaren der Gewerkschaften diese in den Augen der Mitglieder desavouiert. Nur wenn Irrtumer in der Finschåtzung der kủnftigen Ronjunktursituation später rasch ausgeglichen werden können, kann das Klima vermieden werden, das in die stagflation führt.

Ein wesentlicher Aspekt des Vorschlags ist, daB er kurzfristig und bei entsprechender stabilitätspolitik der Regierung auch langfristig die Vollbeschäftigung bei vergleichsweise hoher Preisstabilität sichern kann, ohne daß der im Tarifkonflikt enthaltene Verteilungskampf ausgeschaltet werden muB. Er kann weiter ausgetragen werden, mit gleicher schärfe wie bisher. Nur wird er insofern versachlicht und verbessert, als jetzt mehr um das gestritten werden kann, worum es ja eigentlich geht: den Reallohn. Indem die staatliche Politik 
vermittels dieses Schemas von der unmittelbaren Verantwortung für die Vollbeschäftigung loskommt, kann sie sich darauf konzentrieren, das Preisniveau unabhångig vom Resultat der Tarifauseinandersetzungen zu stabilisieren oder in vorgezelchneten Bahnen wachsen zu lassen. Dann und nur dann entspricht die Nominallohnsteigerung der Reallohnsteigerung. Die Vergangenheit der letzten Jahre hat gezeigt, daß Tarifpolitik einen wesentlichen Elnfluß auf die volkswirtschaftliche Lohnquote haben kann. Auch in der Verteilung der Lohnsumme auf verschiedene Arbeitnehmer-Rategorien bahnen sich wichtige Veränderungen an, die ebenfalls Ergebnis der Tarifpolitik sind. Leider verlief dieser ProzeB in einem Klima verstärkter Inflation, die dazu führte, daB diese wichtigen Verteilungswirkungen im Bewubtsein auch derer, die davon profitierten, wegen der steigenden preise nicht hinreichend registriert wurden. So fühlt sich jeder betrogen, obwohl es fast allen besser geht. Eine Limitierung des durchschnittichen Reallohnzuwachses auf den volkswirtschaftlichen Produktivitåtszuwachs kann folglich nicht Leitschnur der Lohnpolitik werden. Jeder Vorschlag, der darauf hinausläuft, läßt ein wesentliches vertellungspolitisches Element der Lohnbilduna außer Acht. Schon deshalb kann er nicht auf zustimmung der relevanten beteiligten Gruppen rechnen. Andererseits darf diese Ablehnung des Produktivitåtslohngedankens nicht zu der Vorstellung verleiten, daß langfristig der durchschnittliche Reallohn wesentlich stärker steigen könne als die volkswirtschaftliche Produktivität. Rechnet man den impliziten Lohnanteil am Einkommen der Selbståndigen zur Lohnquote hinzu. so liegt diese in der westlichen entwickelten Welt fast durchgängig bei mindestens 80 Prozent des Volkseinkommens. Mehr als 100 Prozent sind nicht zu verteilen. Auf lange sicht ist die Wachstumsrate des durchschnittlichen Reallohns durch das produktivitåtswachstum begrenzt.

Der hier entwickelte Vorschlag hebt ab auf die zahl der Vollzeitbeschäfigten. Nicht mit einbezogen ist die genaue Arbeitszeit der Arbeitnehmer. Uberstunden bleiben auBer Ansatz, womit vermieden werden soll, daB Unternehmen ihrer Beschäftigungsverpflichtung bei Reduktion der Personenzahl durch ưberstundenbezahlung gerecht werden. Aus dem gleichen Grund muß aber gewăhr- 
leistet sein, daß die durchschnittliche Arbeitszeit ohne Ubberstunden in perioden tendenzieller unterbeschäftigung nicht wesentlich unter der durchschnittichen Normalarbeitszeit (ohne Ubberstunden) liegt. Dies kann durch entsprechende Vorschriften ůber die Anrechnung von Beschäftigungsverhältnissen auf die Beschäftigungsverpflichtung garantiert werden. So kann ein Unternehmer seiner Beschäftigungsverpflichtung nicht dadurch nachkommen, daß er Vollzeitarbeitskräfte entläBt und Halbtagskräfte einstellt. Nach oben hin braucht indessen kein Arbeitszeitlimit auferlegt zu werden. Uoberstunden verletzen nicht die pflicht zur Beschränkung der Beschäftigung in Phasen guter Konjunktur. Nur sind sie, wie gesagt, in Phasen schwacher Konjunktur nicht auf die Erfüllung von Beschäfigungsverpflichtungen anwendbar.

Es braucht nicht betont werden, daB das hier vorgeschlagene Instrumentarium ebenfalls angewendet werden kann, um besser mit dem zustrom von ausländischen Arbeitskräften fertigzuwerden.

\section{Fiskalpolitik}

Es war oben schon im einzelnen die Asymmetrie zwischen expansiver und kontraktiver Globalsteuerung diskutiert worden. Bel der Fiskalpolitik ist diese Asymmetrie besonders deutlich geworden, denn hier kommt neben dem beschäftigungspolitischen Aspekt ein haushaltspolitischer dazu. Es ist immer einfacher und populärer. Steuern zu senken und staatsausgaben zu erhöhen, als umgekehrt Steuern zu erhöhen und das Wachstum der Staatsausgaben zu bremsen. Das gilt selbst dann, wenn man die konjunkturelien Effekte der Haushaltsgestaltung auBer Acht läBt. Diese Asymmetrie in der Durchsetzbarkeit expansiver und kontraktiver Flskalpolitik hat dazu geführt, daß man schon zufrieden sein muß, wenn die öffentliche Hand in Phasen der Uberhitzung wenigstens konjunkturneutrale Haushalte beschließt.

In Ländern wie der Bundesrepublik, wo die Zeiten guter Ronjunktur solche schlechter konjunktur quantitativ weit ưberwiegen. werden die Klagen immer stärker, daB die konjunkturpolitisch notwendige Ausgabendämpfung den Ausbau der öffentlichen Infrastruk- 
tur oder wie es weniger technokratisch heiBt, die Lebensqualität gefährde. Sofern staatliche Fiskalpolitik notwendig sei, solle sie durch Variation der steuereinnahmen und nicht so sehr der staatsausgaben getrieben werden. Es ist hier nicht der ort, um zu untersuchen, wie berechtigt das schlagwort vom privaten Reichtum und der offentlichen Armut eigentlich ist. Ich habe melne wweifel. Aber wenn es auch Bereiche offentlicher Verschwendung und Ineffizienz gibt, ist wohl unstrittig, daB gerade wegen der höheren Effizienzsteigerungsraten im Bereich privater Produktion der Anteil der öffentlichen Hand am Sozlalprodukt im langfristigen Trend stelten sollte.

Allerdings glaube ich nicht, daB die Verlagerung der konjunkturellen Fiskalpolitik auf die Einnahmeselte der offentichen Haushalte der stein der weisen 1st. Naturlich soll auch auf der Einnahmeseite Konjunkturpolitik getrieben werden, aber doch nicht ausschlieblich. Eine Umstrukturierung ausgabeorientierter Konjunkturpolitik ist allerdings erforderlich,nicht aber lhre Abschaffung. Es muß ein Grundprinzip der Konjunkturpolitik, insbesondere der Konjunkturdämpfungspolitik sein, an möglichst vielen Stellen anzusetzen, um die Lasten der konjunkturelien Anpassung zu verteilen. Wer die Mo̊glichkeiten der privaten Investition durch restriktive Geldpolitik einschrånt und verteuert, wer die chancen des Exports durch Aufwertung verringert, wer die Kaulkraft der Arbeitnehmerhaushalte durch steuerpolitik reduziert, ist unglaubwirdig ohne eine konjunkturgerechte statsausgabenpolitik. Er verärgert Gewerkschaften, Arbeitnehmer und wåhler und braucht sich nicht zu wundern, wenn es zu "ưberwålzungen" der steuererhöhungen bei den Arbeitnehmern spätestens in der nåchsten Tarlfunde kommt. Im íbrigen sollte eine rational geplante und die steuermittel effizient verwendende Infrastrukturpolitik automatisch konjunkturstabilisierend sein. Dies wird im einzelnen gleich besprochen werden.

Zuerst aber muB auf die Grenzen konjunkturdämpfender steuerpolitik aufmerksam gemacht werden. Natuirlich kann man durch steuerpolitische Maßnahmen versuchen, auf die Investitionstätigkeit Einfluß zu nehmen. Solche Maßnahmen sind dann wirksam, wenn die Investoren 
erwarten können, daß die staatliche konjunkturpolitik bald Erfolg hat und deshalb die zusatzlichen Belastungen von Investititionen in der Hochkonjunktur, bzw. Erleichterungen von Investitionen in der Rezession nur kurz in Kraft sind. Klammert der staat seine Ausgaben aus den Erfordernissen der Ronjunktur aus, so macht er sich moralisch unglaubwirdig. Er kann dann auch wenig 'tun, um die Erfolgschancen seiner Stabilitătspolitik in gůnstigem Licht erscheinen zu lassen. Dann aber werden seine steuerpolitischen Maßnahmen zur Investitionsbeeinflussung wenig bewirken. Reynes hat uns gezelgt, welch zentrale Rolle die Erwartungen der Marktteilnehmer für den Konjunkturverlauf spielen. Ein staat, dessen Konjunkturpolitik moralisch glaubwiridig ist, hat einen viel besseren stand, die Erwartungen der Investoren und Konsumenten in seinem sinne zu beeinflussen als ein stat, dessen rechte und linke Hand so handeln als wiriten sie nicht, was der Mund redet. Die Grenzen der Beeinflussung privaten Konsums durch steuerănderungen sind ebenfalls zu beachten. Bei beschleunigtem Preisauftrieb und guter Beschäfigungslage fuhrt Kaufkraftabschöpfung im Bereich der Masseneinkommen zu Überwälzungsversuchen durch stabilitätswidrige Lohnsteigerungsforderungen. In den höheren Einkommensgruppen fủhrt vorübergehende erhöhte Besteuerung meist nicht zu wesentlichen Einschränkungen des Konsums.

Die Steuerprogression bei der Einkommen- und Lohnsteuer ermöglicht aber eine Form antizyklischer steuerpolitik ohne steuererhöhungen. Wegen der Steuerprogression sind Tarifsenkungen ohnehin dann und wann erforderlich. Es läBt sich nun folgendes Ver-fahren denken. Neben dem Lohn- und Einkommensteuertarif legt der Gesetzgeber einen durchschnittlichen Belastungssatz des zu versteuernden Einkommens fest. Die staatlichen Lohn- und Einkommenssteuereinnahmen stehen den öffentlichen Haushalten nur bis zur Hơhe dieses gesetzlichen durchschnittlichen Belastungssatzes zur Verfügung. Übersteigen die faktischen steuereinnahmen aus der Lohn- und Einkommensstuer - verglichen mit den zu versteuernden Einkommen - diesen durchschittlichen Belastungssatz, so werden sie bei der Zentralbank stillgelegt. Wegen der steuerprogression wira dieser ÜberschuB im verlauf der Zeit gröBer. Bei hierfür geeigneter Konjunkturlage kann nun entweder der Steuertarif nach 
unten oder der gesetzliche durchschnittliche Belastungssatz nach oben angepasst werden. Beides wirkt expansiv: die steuersenkung erhöht die Kaufkraft der privaten Haushalte, die Erhöhung des durchschnittlichen Belastungsgrads vergröBert den Ausgabenspielraum der öffentlichen Haushalte. Der stillzulegende Tell der steuereinnahmen vermindert sich, ja er kann sogar negativ werden, wenn ein hinreichendes Guthaben stillgelegter steuereinnahmen bei der zentralbank vorhanden ist, aus dem das Defizit zwischen gesetzlicher und faktischer Durchschnittsbelastung gedeckt werden kann. Um MiBbrauch auszuschließen, darf die zuführung stillgelegter Steuereinnahmen zu den Haushalten allerdings nie das jeweils vorhandene Gesamtguthaben ůberschreiten.

Diese MaBnahme hat den Sinn, zwelerlei politisch zu erleichtern. Einmal soll sie elner antizyklischen Kaufkraftabschöpfung statt der bisherigen Form eines Beschlusses zur steuererhöhung die Form des Ausbleibens eines Beschlusses zur steuersenkung geben. Sie wird damit leichter durchsetzbar und hat weniger unguinstige Effekte auf die Lohntarifpolitik. Zweitens soll sie die bisherige Tendenz mancher Fisci zur prozyklischen - well einnahmeabhån gigen - Ausgabenpolitik vermindern. Um an den fiskalischen Bonus der steuerprogressionsautomatik heranzukommen, bedarf es eines politisch zu vertretenden expliziten Beschlusses, den in Boomzeiten zu fällen als sünde wider die stabilităt anzuprangern leichter fält als dem Volk zu exklären, weswegen die steuerprom gressionsautomatik zu mangelnder stabilitätsdisziplin des states und der Gemeinden verfürt. Umgekehrt fallen auch expansive steuerliche Maßnahmen in Zeiten schwacher Konjunktur leichter, wenn man weiB, $d a B$ man sie später nicht explizit růckgängig machen mus, was dann ja schwierig sein könnte. Denn durch den progessionseffekt heben sie sich im Verlauf der zeit automatisch auf.

Wir hatten gesagt, daB schon wegen der moralischen Glaubwirdigkeit der Konjunkturpolitik die Ausgabenseite des staatshaushalts nicht ohne Rícksicht auf die Konjunktur gestaltet werden kann. Es gibt aber auch Gründe der fiskalischen Sparsamkeit und Effizienz zugunsten einer konjunkturorientierten Ausgabenpolitik. H1er 1st vor allem von den statichen Infrastrukturinvestitionen die Rede, die 
sich insbesondere auf dem Hoch- und Tiefbausektor auswirken. Es sind dies ưberwiegend langfristige Investitionen, die sich nur über viele Jahre oder Jahrzehnte "amort1sieren." Bel offentlichen Investitionen kann man nicht von elner Amortisation im üblichen sinne sprechen, aber aus der Kosten-Nutzen-Analyse ist der staatichen Verwaltung $z$.T. ja schon heute bekannt, daB man den Nutzen einer Infrastrukturinvestition in Geldåquivalente umrechnet, um entscheiden zu kornnen, ob das Invest1tionsprojekt vorteilhaft ist. Entsprechend kann man im íbertragenen, aber pråzisen Sinne von der Amortisationsperiode eines Infrastrukturprojekts sprechen. Eine U-Bahn, ein Autobahnabschnitt, ein Hochschulgebåude, ein Krankenhaus sind alles Projekte mit einer Amortisationsperiode von anderthalb bis drei oder noch mehr Jahrzehnten.

Wesentliche Kostenfaktoren von Bauinvestitionen mit langer Amortisationsperiode sind nun de Bauprelse und der langfristige Rapitalmarktzinssatz. Diese beiden Rostenfaktoren bewegen sich aber ausgesprochen prozyklisch. Wer wåhrend des Booms baut, baut tever, wer in anderen Phasen des Konjunkturzyklus baut, baut sehr viel billiger. Da die öffentliche Hand auf dem Bausektor eln wesentlicher Nachfrager ist, mußte sie bei hinrelchend konzertiertem Vorgehen ihrer verschiedenen Teile in der Lage sein, eine Art preisfuhrerschaft zu erringen. Steigen die preise furr Bauten zu sehr, so dämpft die offentliche Hand ihre Nachfrage, bei vergleichsweise niedrigen preisen dehnt sie thre Nachfrage aus. Befolgt sie eine solche politik, so wird sie letztlich fur das glelche Geld mehr Bauleistung erhalten, während das håufig zu beobachtende prozyklische Gebaren der öffentlichen Hände die Frage aurkommen läBt, ob die eigentliche Ursache offentlicher Armut viellelcht die offentliche Verschwendung sei.

Sollte ein strukturelles Defizit an Infrastrukturinvestitionen existieren, so ist dessen Beseitigung kein Problem der Konjunkturpolitik, sondern eine Frage der mittel- und langfristigen Finanzplanung. Dann missen eben die steuermittel bereltgestellt werden, um bei mittlerer Konjunkturlage die zusatzlich benötigten Infrastrukturausgaben zu finanzieren. Es ist nicht einzusehen, weshalb mittelfristige Finanzplanung und kurzfxistige Konjunk- 
tursteuerung mittels Ausgabenvariation vom staat nicht kombiniert werden könnte, und zwar so, daB die Infrastruktur nicht zu kurz kommt. 
Anhand. Arbeitsweise eines systems von Preisänderundslizenzen

Um dem Leser den Einstieg in meinen Vorschlag leichter zu machen, habe ich ihn im Text unvollstänig und auch etwas einseitig dargestellt. Der Leser hat heutzutage bei Preisverănderungen insbesondere preiserhöhungen im Kopf, deshalb habe ich es so dargestellt, als ob nur preiserhöhungen unter Lizenz gestellt werden. Das schema kann aber gut nux arbeiten, wenn Preiserhöhungen und Preissenkungen unter Lizenz stehen. Dies wird hoffentich einsichtiq sein, nachdem wir hier einige Detalis des Lizenzsystems geschildert haben.

Es wird eine Clearingstelle fir Prèisanderungslizenzen eingerichtet. Dort hat jede Firma ein Konto fur diese Lizenzen. zu Beqinn eines jeden Ouartals werden auf der Habenseite dieses Kontos Lizenzbeträge eingetragen, deren Höhe sich bemisst nach dem Betrag der Wertschopfung der Firma multipliziert mit dem Prozentsatz der durchschnittichen volkswirtschaftichen preisveränderung, die ihrerseits ja kraft Gesetz bestimmt wird durch die prozentuale verånderung der Geldmenge im vergangenen vierteljahr. Da zum Teil die Daten zur Bestimmung der Lizenzbeträge noch nicht denau vorliegen dürten, wenn das vergangene ouartal gerade exst abgelaufen ist, kann es notwendig sein, nach einem honat noch elne Korrektur bei dieser Eintragung der Lizenzen vorzunehmen. oder man kann diese Korrekturen bei den Eintragungen nach einem weiteren Vierteljahr berůcksichtigen. Wichtig ist zu sehen, daß das Lizenzkonto im Extremfall einer neativen volkswirtschaftichen Preisainderungsrate durch eine entsprechende Eintragung auf der sollselte belastet werden kann. Durch diese vierteljährlichen staatlicherseits vorgenommenen Elntragungen entstehen also "Guthaben" bww. "Verpelichtungen" der Firmen auf ihrem Lizenzkonto. Eine Firma meldet nun die von ihr vorgenommenen Preisănderungen an. Diese Preisånderungen schlagen sich in entsprechenden Eintragungen auf dem Lizenzkonto nieder, und zwar eine Preiserhöhung auf der Sollseite und eine Preissenkung auf der Habenseite. Die Hỏhe der Buchung ist proportional zum Prozentsatz der preisånderung sowie natuirlich proportional zum Umsatz des produkts, um dessen Preis es geht. Die Recheneinheit der Iizenzkonten istalso 
"ein Prozent Preiserhöhung einer DM Quartalsumsatz". Hat ein produkt einen vierteljăhrlichen Umsatz von 600.000 DM und wird sein preis um 4 prozent erhöht, dann werden auf der Soliseite 2.400.000 Lizenzeinheiten gebucht. Wird stattdessen der Preis dieses produktes um 2 prozent gesenkt, dann gibt es eine Buchung auf der Habenseite von 1.200.000 Lizenzeinheiten. Wirdder Preis eines Vorprodukts von dem Lieferanten erhöht, so schlägt sich das in einer Buchung auf der Habenselte unserer Firma nieder, so wie es zu einer entsprechenden Buchung auf der soliseite bei dem Lieferanten kommt.

Schliesslich gibt es, wie schon gesagt, die Mögllchkelt des Handels in Preisänderungslizenzen. Eine Firma kann also Preisänderungslizenzen an eine andere übertragen. Wle wir gleich sehen werden, ist es nitzlich, einen offiziellen Tageskurs firr Preisänderunaslizenzen zur Verfügung zu haben. Schon deshalb ist es sinnvoll, einen amtlichen Makler für die Preisånderungslizenz einzusetzen. Es ist wichtig zu sehen, daß der frele Marktpreis fir Preisanderungsizenzen nicht notwendigerweise positiv sein muss. Er kann auch Null oder negativ sein. Ist er positiv, dann sind die Firmen froh uber einen hohen Habensaldo an Lizenzen, die sie auf der Börse verkaufen können. Ist der preis fur Preisänderungslizenzen negativ, dann sind die firmen froh uber einen hohen Sollsaldo auf dem Lizenzkonto, den sie nun anderen gegen Bezahlung abtreten können. Negative Preise fur Preisänderungslizenzen können z.B. dann auftreten, wenn die Geldmenge stark erhöht wird, so daß gemäß gesetzlicher vorschrift die Gutschriften auf den Lizenzkonten der Firmen hoch sind. Verpflichtet man die Firmen, nicht nur sollsalden sondern auch Habensalden auf ihren Lizenzkonten zưiq abzubauen, dann kann dieser negative Preis firr Iizenzen entstehen.

Eine Verpflichtung zum Saldenabbau ist natuirlich notwendig, damit von den Lizenzen überhaupt ein Einfluss auf die Preisbildung ausgeht Sie kann die folgende Form annehmen. Jeder Saldenuberschuss zum Quartalsende, der im Verhåltnis zur Wertschöpfung der Firma einen bestimmten Betraq übersteiat, wird qegen Erhebung einer Gebihr vom stat übernommen. Diese Gebihr ist fuir jede uibernommene Lizenzeinheit das Zehnfache des Preises der Lizenzeinheit beim öffentlichen Makler eine woche vor Ende des Duartals. 
Tendiert z.B. die Lizenz dazu, derzeit einen positiven Preis zu haben, dann ist es fur eine Firma mit einem den "Freibetrag" ubbersteigenden sollsaldo ginstiger, durch zukauf von prelserhoihungsizenzen eine woche vor dem ouartal diesen sollsaldo abzubauen als ihn stehen zu lassen und ihn durch den staat abdecken zu lassen. Wir legen den stichtag fur den relevanten Marktpreis eine woche vor das Quartal, so daB die Firmen in der letzten woche des ourtals noch Gelegenheit haben, ihre Preise zu ändern und auf diese Weise ihre Lizenzsaldos auszugleichen. Ein Missbrauch des systems ist naturlich dadurch möglich, daß Firmen ihren Preis kurz vor ouartalsende senken und ihn gleich danach wieder erhöhen. Dieser Missbrauch kann aber durch entsprechende Vorschriften lelcht ausgeschaltet werden. Beispielsweise kann das prinzip eingefuhrt werden, daB eine Preisänderung je nachdem mehr oder weniger tizenzen erfordert, wann sie im Quartal durchgefuhrt worden ist, so daß praktisch die durchschnittlich während des ouartals guiltigen Prelse im Verhältnis zu den durchschnittich im vorausgegangenen ouartal gültigen Preisen der lizenzrelevante Tatbestand sind. In diesem Fall muss es aber möglich sein, sozusagen durch Lizenz stundung einen vorgriff auf die Lizenzen des năchsten ouatals dann zu erreichen, wenn man in der letzten woche des Quartals die Preise entsprechend ändert. Fine solche Preisanderung zum ouartalsende wirde dann so behandelt, als sel sie schon zu ouartalsbeqinn exfolgt, wobei aber dann im nächsten ouartal eine entsprechende Fintragung auf dem Konto das Faktum ausqleicht, daß faktisch die preisänderung viel spăter exfolgt ist. Bei hohen Marktpreisen für Iizenzen wird man dann jeweils zum ouartalsende mit Preissenkungen rechnen können. Analog dem Vorgehen bei der Umsatzsteuer (Mehrwertsteuer) sollten beim Preisänderungslizenzverfahren die Exporte und Importe behandelt werden. Exporte werden von der inländischen Umsatzsteuer an der Grenze entlastet. Importe werden mit der inlänischen Umsatzsteuer belastet. Entsprechend sollten die Exporte nicht dem Preisănderungsilienzverfahren unterliegen, wăhrend umgekehrt Ånderungen der Importpreise nicht lizenzfrei auf Anderungen beim Wiederverkaufspreis des Importeurs angerechnet werden. Auf diese Weise besteht auch nicht die Notwendiakeit im Ausland durchgefinrte Transaktionen zu überprůfen. 
Das problem neuer produkte händt zusammen mit den Problemen der Ermittluna richtiger Preisindizes. Es muss garantiert sein, dab sich aus der Behandlung neuer produkte in diesem Lizenzverfahren keine Diskrepanz zwischen dem statistisch ermittelten Preisindex und dem volkswirtschaftlichen preisniveau ergibt, das diesem Verfahren entspricht.Das ist nicht nur ein Problem der neuen produkte, sondern ebenso eines des statistisch ermittelten Preisindex. Das prinzip muss sein: wird ein neues Produkt mit einem solchen Preis einqefinrt, daß dieser Preis keinen Effekt nach oben oder unten auf den statistisch gemessenen Preisindex hat, dann benötigt das neue Produkt bei seiner Einführung keine Preisänderungslizenz. Wird es zu einem höheren preis eingeführt, dann wird die Firma, die es einfuhrt auf der soliseite der preisänderunaslizenzen mit einem bestimmten Betraq belastet, über dessen Höhe gleich etwas gesagt werden wird. Umgekehrt erhält die Firma eine Eintragung auf der Habenseite, wenn der preis so ist, daß er einen dämpfenden Effekt auf den volkswirtschaftlichen Preisindex hat. Die genaue Hoohe der Eintragung auf dem Preisånderungslizenzkonto kann zum zeltpunkt der Einfuihrung des Produkts nicht festgestellt werden. Sie richtet sich sinnvollerweise nach dem Umsatz des produkts in den ersten zwei oder drei Jahren seines Vertriebs. Natuirlich ist damit längst nicht alles gesagt ưber dieses Thema. Insbesondere könnten die heute verwendeten Preisindizes, 2.B. des Sozialprodukts, nicht befriedigend sein, wenn man sie in dieser Weise zur Lo̊suna des Problems neuer Produkte beim Preisånderungslinzenzverfahren einsetzt. Möqlicherweise missten diese Indizes methodisch und empirisch auf eine neue Basis gestellt werden, um hierfür zu taugen. Das mag aber ohnehin winschenswert sein. Das Problem der Messung des Lebensstandards und seiner Entwicklung bei ständiger Weiterentwickluna der Produktpalette ist ohnehin wichtig und aktuell. Wenn auch solche Detallprobleme wichtig und interessant sind, so sollte man ihre Bedeutung auch nicht überschätzen. Approximative, pragmatische Lösungen sind $m . E$. 
durchaus hinreichend für eln befriedioendes Funktionieren des Gesamtsystems, das hier vorgeschlagen wird. Der Grund hierfur liegt darin, daß die Marktpreise furr die Preisainderungsizenzen im Durchschnitt nicht sehr hoch sein werden, so daß Fehler des systems im Detail keine stark verzerrende Wirkung auf die unternehmerischen Entscheidungen haben können. Um zu begrůnden, daß die Preise der Lizenzen relativ niedrig sein werden, müssen wir die Dynamik des vorgeschlagenen systems diskutieren.

Geht man einmal davon aus, daß Im Trend das Verhåltnis "Sozialprodukt zu volkswirtschaftliche Geldmenge" (= Umlaufgeschwindigkeit des Geldes) konstant bleibt und nur saisonal bzw. konjunkturell schwankt, so muss auf långere sicht das Preisniveau des Sozialprodukts sich genau nach der Formel entwickeln, nach der in unserem preislenkunassystem die zugelassenen volkswirtschaftliche preisånderungsrate in Abhångigkeit der Geldmenqenverånderung berechnet wird. Långerfristig arbeitet unser system also gar nicht gegen den Markt. Die Preise wirden sich ohne das system bei oleicher Geldversorgung mittelfristia qenau so entwickeln wie mit dem system. Das einzige, was das system beeinflusst, ist das genaue "Timing" von preisänderungen. Deshalb ist der Preis fur Preisänderungslizenzen im Durchschnitt Nul1. Bel den bekannten wirkungsverzögerungen von Geldmengenänderungen wird ein "zyklus" für die Lizenzpreise wenige Jahre (3-5) nicht ủbersteigen können. Wenn man das weiss, wird man aber nicht allzu hohe Prelse fur diese Lizenzen zu zahlen bereit sein.

Um die zu erwartenden Grössenordnungen der Preise für Preisänderungslizenzen genau abzuschätzen, wollen wir ein einfaches Berechnungsmodell diskutieren, wobei aber drei Dinge vorweg klargestellt werden sollen. Erstens muss gesagt werden, welche Maßeinheit die Marktpreise für Preisånderungsilienzen eigentlich haben. Zweitens muss der Begriff des "Monopolgrads" erläutert werden, der im Modell eine Rolle spielt. Drittens muss noch etwas 
zum Problem des Risikos auf den Märkten für diese Lizenzen gesagt werden. Eine Lizenzeinheit ermåchtigt zur einprozentigen einmaligen Erhöhung des Preises einer ware mit 1 DM vierteljåhrlichem Umsatz. Die Lizenzen zur Preiserhöhung werden damit gemessen in Prozent mal Umsatz(DM) "haben also

$$
\text { Zeit(vierteljahr) }
$$

bis auf den dimensionslosen Faktor $\frac{1}{100}$ (=Prozent)

dieselbe Dimension wie der Umsatz pro Zeiteinheit. Der Preis eines objekts ist die Menge Gelde (DM), die pro Quantitätseinheit des objekts bezahlt werden muss. Wird die Quantităt z.B. in ka gemessen, hat dex Preis die Dimension $\frac{\mathrm{DM}}{\mathrm{kg}}$.

Hier, bei den Preisänderungslizenzen, hat das objekt die Dimension DM Zeiteinheiten also hat der preis die Dimension $\frac{\mathrm{DM}}{\mathrm{DM}}$ Zeiteinheiten: er hat also einfach die Dimension "Zeit". Der Preis der Preiserhöhungslizenz mit der Dimension "Zeit" gibt die periode an, innerhalb derer ein Unternehmen die Kosten des Kaufs der Preiserhöhungslizenz durch die dadurch ermöglichte Preiserhöhung ertragsmässid wieder ausqleichen kann, vorausgesetzt, daß sich der Absatz durch die Preiserhöhung nicht ändert. Es ist bei der Abschätzung der preise der Lizenzen angesichts ihrer Dimension "Zeit" klar, daß die Preise wesentlich durch die Länge des "zyklus" bestimmt werden, den die Preise fur preisänderungslizenzen durchlaufen.

Als nächstes ist dex "Monopolgrad" zu diskutieren. Die traditionelle Theorie des Marktes verwendet diesen Begriff, der durch das Verhältnis von Marktoreis und Grenzkosten der Produkte definiert ist. Der Name "Monopolgrad" suggeriert, daß diese Messgrösse umso höher lieat, je stärker das wirtschaftliche Geschehen von grossen Konzernen bestimnt wird. Dies ist M.E. aber irrefuhrend. Gerade viele sehr kleine Betriebe des Dienstleistungsbereiches sind durch einen sehr hohen Monopolgrad gekennzeichnet. Als Beispiel diene der Friseurladen an der Ecke. Abgesehen von einigen fixen Kosten der Ladenmiete und der Amortisation von Geräten bestehen 
seine Kosten weitgehend aus Lohnkosten. Diese Lohnkosten sind aber auf kurze sicht fix: sie hången unterhalb der zahl maximal bedienbarer Kunden nicht von der Zahl der Kunden ab. Bei fixem Salär des Friseurgesellen (bzw. bei einem zugerechneten fixen Salär firr den Inhaber, wenn er selbst bedient) sind fir den Friseurbetrieb die Grenzkosten der Bedienung des einzelnen Kunden nahezu Null. Der Monopolgrad des Friseurbetriebs ist sehr hoch, viel höher als der Monopolarad eines grossen Stahl-oder Automobilwerks. Da sich aber der Name "Monopolarad" eincebiorert hat, der Begriff sehr häufig verwendet wird und in vielen zusammenhängen wichtig ist, wollen wir es bei dieser Benennung lassen. Nur hat dieser Monopolorad nichts mit dem Begriff wirtschafticher Macht zu tun. Es stellt sich heraus, daß der spielraum fur das "Timing" von Preisänderungen umso grösser ist, je höher der Monopolgrad einer Firma ist. Dabei muss man im Sinne unseres Vorschlags aber auf den "Nettomonopolgrad" achten, worunter zu verstehen ist:

Wertschöpfung

Variable kosten abzüglich Vorleistung, die in den variablen Kosten enthalten sind

minus 1. Der Nettomonopolgrad gibt also an, um wieviel die qesamte Wertschöpfung eines Betriebs höher ist als der Teil der Wertschöpfund, der den variablen Kosten zuzurechnen ist. Dabei sind die variablen Kosten hier ganz eng zu fassen als die Kosten, die anfallen, um zusätzliche produkteinheiten/oder Leistungseinheiten kurzfristia zu erbringen. In den meisten konsumentennahen Dienstleistungsbetrieben (Einzelhandel, Bankwesen, Wohnungsvermietung, Hotels, Restaurants, Personenbeforderung etc.) ist dieser Nettomonopolarad recht hoch. Dies ist im übrigen auch einer der Gründe, weshalb die Konsumentenpreise immer spät auf die Konjunkturentwicklung reagieren. Im zusammenhang mit unserem Vorschlag bedeutet diese Beobachtung, daß sein Effekt auf die Preisbildung gerade im konsumentennahen Bereich stark sein wird, etwas, was anqesichts der Rückwirkung der Konsumentenpreise auf die Nominallohnbildung besonders begri̊st werden kann. Man kann erwarten, daß die konsumnahmen Dienstleistungsbetriebe - etwa der Einzelhandel - bei positivem Marktpreis der Preiserhöhungsizenz 
einen Teil der Preiserhöhung ihrer Lieferanten auffangen und nicht sofort an die Konsumenten weitergeben, also sozusagen als preispuffer fungiexen. Dies natürlich nur vorubergehend: sie werden, schon um auf die Dauer auf ihre fixen Kosten zu kommen, im Lauf der Zeit diese Preiserhöhungen zu überwålzen haben.

Als drittes ist noch einmal auf das Problem des Risikos betreffend die künftigen preise für Preisänderungslizenzen hinzuweisen. Wenn Terminmärkte fur Preisänderungslizenzen existieren, dann läßt sich ein grosser Teil dieses Risikos abdecken. Wegen der Kettund der Preisănderungslizenzen an die Geldmengenveränderung ist zu erwarten, daB das Bedirfnis, hinter den dekretierten volkswirtschaftichen preissteigerungsxaten zurickzubleiben, mittelfristig qenau so stark ist, wie das Bedürnis, diese preissteiqerunqsraten zu ỉbertreffen. Aus diesem Grunde sollte es auf beiden seiten der Terminmärkte ungefăhr gleich viele partner aeben, die durch Kauf bzw. Verkauf der Lizenzen, sich und ihren Marktpartnern das Risiko verkleinern. Bei einer gewissen Nachhilfe seitens öffentlicher stelien kann also mit dem Funktionieren dieser Risikoausschaltung gerechnet werden. Wir gehen deshalb im folgenden Modell von einer Situation aus, in der die einzelne Firma Sicherheit daruber hat, welche Preise kůnftid für Preisänderungslizenzen zu zahlen sind.

Nun das Modell. Es läßt sich leichter formulieren, wenn wir kontinuierliche Zeit einfuhren. Es sei $\lambda(t)$ der Marktpreis für Preisänderungslizenzen. Da es uns nur auf eine approximative Analyse ankommt, wollen wir zur Erleichterung des Rechenqanges ganz einfache Annahmen machen. Es sei also $\lambda(t)$ stückweise linear in folqender Weise:

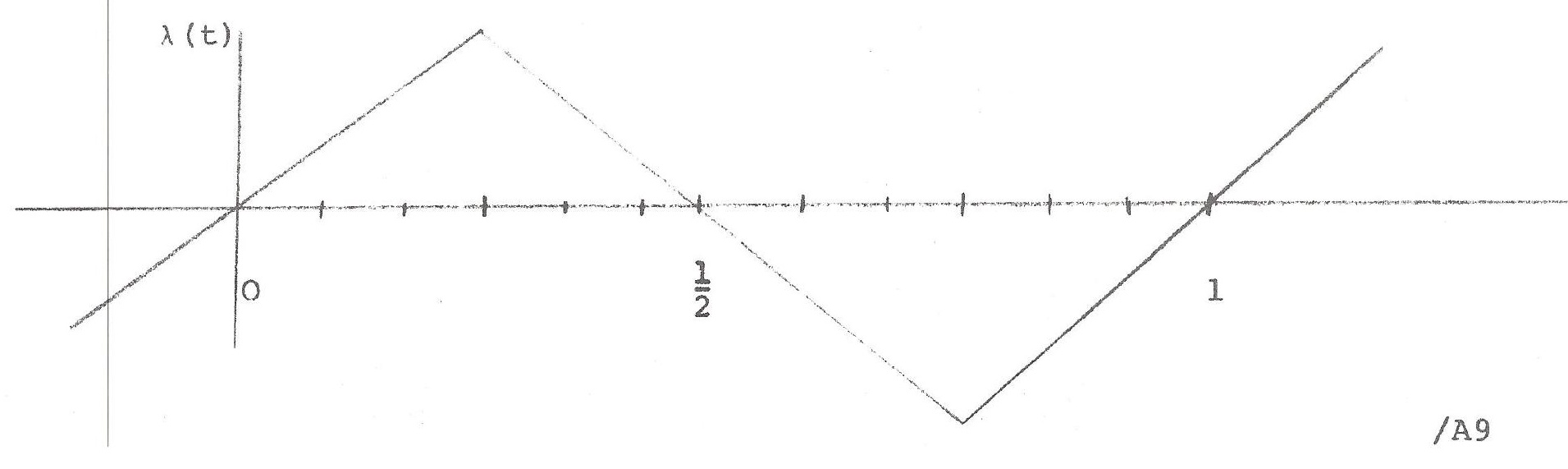


Der zyklus von $\lambda$ daure also eine periode (dies ist nur eine Konvention iber die Messun der zelt. Zur zeit $t=0$ erreicht $\lambda$ seinen maximalen wert $\lambda *$, zur zeit $t=\frac{1}{2}$ entsprechend seinen niedrigsten Wert $-\lambda *$. Es sei nun eine Firma gegeben, die ohne Preisänderungslizenz ihren Preis einfach konstant bei $\overline{0}$ halten wirirde. (Das Modell lässt sich leicht auf Fälle übertragen, wo der Preis mit der Zeit wächst oder sinkt). Da sie nun aber von der Möglichkeit, an den Preisänderungslizenzen zu verdienen, Gebrauch machen will, ändert sie ihren Preis im Lauf der zeit und zwar so, daß ${ }^{\circ}$, die zeitliche Veränderungsrate negativ ist, wenn $\lambda(t)$ positiv ist, und umgekehrt positiv ist, wenn $\lambda(t)$ negativ ist. Zur Vereinfachung sei p stückweise linear angenommen. Der maximale Wert von $p$ sei $p_{\max }=\bar{p}+h$ der minimale wert sei entsprechend $p_{\min }=\bar{p}-\mathrm{h}$. Ex ist dann $\mathrm{p}$ als Funktion der zeit

$p(t)=\bar{p}+h-4 h t \quad$ furr $0 \leq t \leq \frac{1}{2}$

$p(t)=\bar{p}+h-4 h(1-t)$ furr $\frac{1}{2} \leq t \leq 1$

Die Frage ist nun: welches ist der fir die Firma optimale wert von h, d.h. der maximalen Preisabweichung vom "Normalpreis" $\bar{p}$ ? Der Ertraq auf dem Markt fïr Preisånderungslizenzen zu einem bestimmten zeitpunkt ist geqeben durch

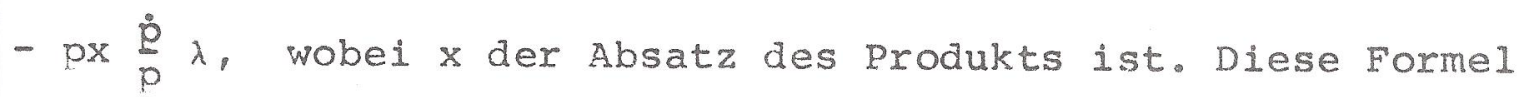
erklärt sich wie folgt:

$\lambda$ ist der zu zahlende preis fir eine proportionale preiserhohung pro Umsatzeinheit. Dies multipliziert mit der Rate der Preiserhöhung $\frac{\text { L }}{p}$ und dem Umsatz px erqibt die Gesamtkosten der Preisänderung. Der Gewinn ist der neqative Betrag davon. Zur Vereinfachung der Rechnung ersetzen wir in dieser Formel den Absatz $x$ durch den durchschnittlichen Absatz $\bar{x}$, dex eine zeitliche Konstante ist. So lautet die Gewinnfunktion jetzt

$$
-\overline{\mathrm{x}} \mathrm{p} \frac{\mathrm{p}}{\mathrm{p}} \lambda=-\overline{\mathrm{x}} \mathrm{\rho} \lambda
$$

Diese Manipulation ändert nichts Wesentliches am Ergebnis. 


\section{A 10}

Nun ist $\dot{p}=\frac{d p}{d t}$ aleich $-4 h$, wenn $\lambda(t)$ positiv ist und gleich $+4 h$, wenn $\lambda(t)$ negativ ist, also erhalten wir $-\bar{x} \dot{p}(t) \lambda(t)=\bar{x} 4 h|\lambda(t)|$, wobei $|\lambda(t)|$ der Absolutbetrag von $\lambda(t)$ ist. Der durchschnittliche Ertrag auf dem Lizenzmarkt ist deshalb gleich $\bar{x} 4 \mathrm{~h}$ mal dem durchschnittlichen wert von $|\lambda(t)|$. Bei der linearen struktur von $\lambda(t)$ ist dieser Durchschnittswert von $|\lambda(1)|$ gleich der Hälfte des Maximalwerts $\lambda *$. Bezeichnen wir ihn mit $\bar{\lambda}$, so ist also der Ertrag auf dem Lizenzmarkt oro zyklus gegeben durch $4 \bar{x} h \bar{\lambda}$. Ist es dann nicht guinstig, h so gross wie möglich zu machen? Nein, denn die Abweichung des Marktpreises vom Normalpreis $\bar{p}$ bedeutet, daß die Gewinne auf dem Produktmarkt der Firma vermindert werden.Diese Gewinnschmålerung durch Abweichungen vom optimalen Preis können, zumindest fuir nicht allzugroße Abweichungen durch eine quadratische Funktion approximiert werden. Sei der Marktverlust $V$, so setzen wir an

$$
V=\bar{p} \bar{x} \text { a }\left(\frac{p}{\bar{p}}-1\right)^{2}
$$

Im Fall des klassischen Monopols mit linearer Nachfrage-und Rostenfunktion stelit sich beispielsweise heraus, daB der Proportionalitäsfaktor a bestimmt wird durch den Monopolorad $\mu$ entsprechend der Forme1 $\quad \mathrm{a}=\frac{1+\mu}{\mu}=\frac{1}{\mu}+1$

Man sieht, daß der relative Verlust (Verlust bezogen auf den Normalumsatz) bei einem von $\bar{p}$ um einen bestimmten prozentsatz abweichenden Preis umso arösser ist, je kleiner der Monopolgrad. Kennt man den Wert von a, so kann man den durchschnittichen veriust berechnen, der auf dem Produktmarkt im Verlauf eines zyklus entsteht. Der Preis $p$ ist im Verlauf des zyklus gleichmässig verteilt in dem Intervall zwischen $\bar{p}-h$ und $\bar{p}+h$. Entsprechend ist $\frac{p}{\overline{0}}$ gleich verteilt im Intervalivon $1-\frac{h}{\bar{p}}$ bis $1+\frac{h}{\bar{p}}$. Da der verlust eine

quadratische Funktion der Abweichung $\frac{p}{\bar{D}}$ vom Mittelwert 1 ist, ist der mittlere Verlust gleich der Varianz der Verteilung von $\frac{p}{\bar{p}}$ 


\section{A 11}

multipliziert mit $\bar{p} \bar{x}$ a. Die Varlanz der genannten Gleichverteilung ist aber $\frac{1}{3}\left(\frac{h}{p}\right)^{2}$, so daß der verlust fur den ganzen zyklus nunmehr $\vec{v}=\bar{p} \bar{x}$ a $\frac{1}{3}\left(\frac{h}{p}\right)^{2}$

beträgt. Saldiert man dies qegen den Gewinn aus den Operationen auf dem Markt fưr Preisänderunaslizenzen, so erhålt man

$$
\bar{G}=4 \bar{x} \bar{\lambda} h-\frac{1}{3} \bar{p} \bar{x} a\left(\frac{h}{\bar{p}}\right)^{2}
$$

Differenzierung dieses Ausdrucks nach h fihrt nach Nullsetzung zu folgender Bedingung fir das optimum

$\frac{\mathrm{d} \overline{\mathrm{G}}}{\mathrm{dh}}=4 \overline{\mathrm{x}} \bar{\lambda}-\frac{2}{3} \frac{\overline{\mathrm{x}}}{\overline{\mathrm{p}}} \mathrm{a} \mathrm{h}=0$

oder

$h=6 \frac{\bar{p} \bar{\lambda}}{a}$ oder $\frac{h}{\bar{p}}=6 \frac{\bar{\lambda}}{a}$

Wir erinnern uns, Caß $x^{*}$, der maximale wert von $\lambda(t)$, doppelt so groß ist wie $\bar{\lambda}$, so daß wir auch schretben können:

$$
\frac{h}{\bar{p}}=3 \frac{\lambda *}{\mathrm{a}}
$$

Jetzt ist es wichtia, sich ein Bild von der Grössenordnung von a zu machen. Als Anhaltspunkt wählen wir den Monopolisierungsgrad, da $a=\frac{1}{\mu}+1$ gilt. Setzen wir dies in die Formel ein, erhalten wir

$$
\frac{h}{\bar{p}}=3 \frac{\lambda *}{\frac{\mu+I}{\mu}}=3 \lambda * \frac{\mu}{1+\mu}
$$


Ein durchschnitticher Monopolisierungsgrad von $\mu=1$ scheint uns nicht zu hoch gegriffen. Dies beaeutet, daß im Durchschnitt 50 Prozent der wertschöpfung der Volkswirtschaft unter dem hier zu betrachtenden Gesichtspunkt als fixe. Kostenbestandteile anzusehen sind. Das ist plausibel, wenn man insbesondere bedenkt, welch großer Teil der Lohnsumme heute fur Arbeitsleistungen bezahlt werden, die aus funktionalen oder sozialen Grinden unabhängig davon erbracht werden, ob kurzfristig die Nachfrage nach den produkten absinkt oder nicht. "Kurzfristig" heißt hier im Verlauf einer Zeitspanne, in der die preise des Produkts nicht revidiert werden. Vielleicht ist $\mu$ sogar höher als eins. Rechnet man aber vorsichtig mit $\mu=1$, so erhält man

$$
\frac{h}{\bar{p}}=\frac{3}{2} \lambda^{*}
$$

Diese Formel besagt folgendes: die maximale lizenzinduzierte prozentuale Preisabweichung ist anderthalbmal so groß wie der maximale Preis für Preisänderungslizenzen, wenn man in solchen Zeiteinheiten mißt, daß der zyklus von $\lambda(t)$ eine Periode lang ist. Faktisch wird der zyklus von $\lambda(t)$ ungefähr dem Konjunkturzyklus entsprechen - wie gleich noch ausfuhrlicher diskutiert werden wird. setzen wir diesen zyklus einmal mit einer Länge von vier Jahren an, so wäre der wert von $\lambda^{*}$, ausgedrückt in Jahren, mit 4 zu multiplizieren. In diesen zeiteinheiten wäre dann

$$
\frac{h}{b}=\frac{3}{8} \quad \lambda^{2}
$$

Nun kann es unmöglich das ziel der Konjunkturpolitik sein, die maximale lizenzinduzierte Preisabweichung gröBer als lo Prozent, oder allenfalls 12,5 prozent zu machen. Ein solcher spielraum reicht für eine vernünftige konjunkturpolitik, für die Beeinflussung von Tarifverhandlungen im sinne gröBerer stabilität völlig aus. Damit können wir die linke seite gleich 12,5 prozenz = $\frac{1}{8}$ setzen und dies ergibt einen wert für $\lambda^{*}$ von $\frac{1}{3}$, gemessen in Jahren $\overline{8}$ oder von 4 Monaten. Der maximale Preis für $\overline{3}$ Preiserhöhungslizenzen ist somit 4 Monate. 
Das bedeutet: die Firmen müssen nie soviel für preiserhöhungslizenzen bezahlen, als daß sie nicht binnen weniger Monate fähig wären, die Kosten der Preiserhöhung durch eben diese Preiserhöhung wieder wettzumachen.

Bei der hier aufgemachten Rechnung ging es nur um eine GröBenabschätzung, die ihrerseits Beweis dafir sein soll, daB Probleme der illegalen oder legalen Umgehung des Lizenzsystems oder der Verzerrung des Wirtschaftsprozesses durch das Lizenzsystem angesichts des niedrigen Preises der Lizenzen nicht allzuschwer zu nehmen sind. Dies wäre natürlich anders, wenn die preise für Preisänderungslizenzen sehr hoch wären. Es muß noch hinzugefügt werden, daB die situation noch günstiger aussieht fuir den Fall, daß aie Firmen eine noch bessere Politik verfolgen als wir es hier vorausgesetzt haben.

Natürlich könnte es sein, daß die Umlaufgeschwindigkeit des Geldes nicht nur saisonal und konjunturell sondern auch langfristig nicht genau konstant ist. Wird in der Formel zur Regulierung der Preiserhöhungen im Lizenzsystem die langfristige Ronstanz der Umlaufgeschwindigkeit vorausgesetzt, so könnten sich in diesem Fall Schwierigkeiten ergeben. Aus diesem Grund muß die Formel hinreichend anpassungsfähig sein. Dies kann dadurch erreicht werden, daß man einen Korrekturterm dazufügt, der von einem gewogenen Durchschnitt vergangener Preise für Preisånderungslizenzen abhängt. Es sei $\lambda$ dieser gewogene Durchschnitt früherer Preise für Preisänderungslizenzen. Es sei $x$ die (wieder durch geeignete Durchschnittsbildung vergangener Werte $\mathrm{zu}$ bestimmende) vierteljährliche Trendwachstumsrate des realen Sozialprodukts, es sei m die Wachstumsrate der Geldmenge im vergangenen Quartal und sei $\pi$ die volkswirtschaftlich erlaubte Preissteigerungsrate in diesem quartal. Dann lautet die Formel bisher

$$
\pi=m-x
$$

Wir modifizieren sie nun zu

$$
\pi=m-x+\alpha \lambda
$$


wobel a eine positive Konstante ist. Dadurch übt das System einen stabilisierenden Einfluß auf die preise für Preisänderungslizenzen aus. Waren diese in der Vergangenheit im Durchschnitt positiv, so führt dies dazu, daß heute mehr Preiserhöhungslizenzen vergeben worden als andernfalls.Bei geeigneter Durchschnittsbildung - d.h. uber 4 oder 5 Jahre hinweg - und bei langfristiger Konstanz der Umlaufgeschwindigkeit des Geldes kann allerdings dieser Durchschnitt nur unwesentlich von Null abweichen. In diesem Fall ist die ursprüngliche Formel approximativ gültig. Hat die Umlaufgeschwindigkeit eine Tendenz zu fallen, so daß im Zeitablauf eine höhere Geldmenge für ein gegebenes nominelles sozialprodukt exforderlich ist, so hätten die Firmen es vorgezogen, ihre preise weniger zu erhöhen als sie es im Iizenzsystem im Durchschnitt mußten, dann wäre $\lambda$, der Durchschnitt der Lizenzpreise, negativ und $\pi$ wäre in der Tat kleiner als andernfalls. Umgekehrt ist bei einer Tendenz der Umlaufgeschwindigkeit zu steigen der Durchschnittswert $\lambda$ positiv, und daher ist $\pi$, die Preiserhöhungsrate bei gegebenem $m$ und $x$ höher als bei im Trend konstanter Umlaufgeschwindigkeit.

Es ist schon angedeutet worden, daß der zyklus der Lizenzpreise ungefähr dem Konjunkturzyklus entspricht. Dieser hat seit dem zweiten Weltkrieg eine Länge von 3 bis 5 Jahren. Man kann aus der Kenntnis der Preisniveauentwicklung im Konjunkturzyklus Schlüsse auf die Preise der Preisänderungslizenzen ziehen. In der Rezession ist die Umlaufgeschwindigkeit des Geldes kleiner als in der Hochkonjunktur. Wenn die Umlaufgeschwindigkeit einen Wert unterhalb ihres Trendwerts annimmt, wenn also das sozialprodukt im Verhältnis zur Geldmenge gemessen an längerfristigen Maßstäben zu klein ist, kann sich das darin äussern, daß das reale sozialprodukt unter seinem Trendwert liegt, und es kann darin zum Ausdruck kommen, daß das Preisniveau niedriger ist als der Trendwert. Heutzutage beobachtet man, daß primär das reale Sozialprodukt betroffen ist und erst $z u$ einer späteren Phase das Preisniveau. Das Lizenzsystem hat die Wirkung, das Preisniveau stärker an seinem Trendwert zu halten, so daß im Verlauf einer Rezession die Schere zwischen dem "natürlichen" Preisniveau und dem Preisniveau mit dem Lizenzsystem grösser wird. Die Unternehmen werden durch das Lizenzsystem veranlasst, im Verlauf der Rezession zunehmend höhere Preise zu 
fordern als sie es ohne Lizenzsystem getan häten. In der ganzen rezessiven Phase werden die Preisänderungslizenzen elnen vergleichsweise hohen negativen Preis haben. Kommt dann der Aufschwung, dann erhöht sich die Umlaufgeschwindigkeit des Geldes, wiederum aber primär aufgrund eines starken realen Wachstums, während sich im Bereich der preise vorerst nicht viel tut. Die natuirlichen Preise bleiben vorerst unter dem Trend, die Diskrepanz zu den Lizenzpreisen bleibt bestehen. Andererseits wird der Preis für Preiserhöhungslizenzen steigen, d.h. zuerst sein hohes negatives Niveau abbauen und schliesslich positiv werden. Zwar sinkt damit an sich der Anreiz, die positive Diskrepanz zwischen effektiven (lizenzbeeinflußten) und natürlichen Preisen aufrechtzuerhalten oder gar zu vergro̊ssern. Dieser Tendenz der Angleichung von effektiven und natürlichen Preisen wird aber nicht nachgegeben, da man steigende Preise für Preisänderungsiizenzen erwartet, die es sinnvoll macht, mit Preisånderungen nach unten (relativ zum Trend) noch abzuwarten. Erst im späteren verlauf des Aufschwungs findet eine gewisse Anpassung statt, die aber natirlich die Form steigender natuirlicher Preise annimmt bei konstanten lizenzinduzierten effektiven Preisen. Die Boomphase ist in ihrem früheren statium dadurch gekennzeichnet, daß die natürlichen Preise kaum über ihrem Trendwert liegen, daher eine Diskrepanz zwischen ihnen und den effektiven Preisen nicht vorhanden ist. Jedoch haben die preise für Preisånderungslizenzen nun ein hohes positives Plateau erreicht. Im Verlauf des Booms würden nun die natürlichen Preise, ihren Trendwert immer stärker übersteigen: die Diskrepanz zwischen effektiven und natůrlichen Preisen wird zunehmend negativer, da die Firmen angesichts der hohen Preise für Preisänderungslizenzen auf Preiserhöhungen verzichten. Gegen Ende des Booms ist die Diskrepanz am höchsten. In der Phase des Abschwungs sinkt der Preis fủr Preiserhöhungslizenzen und wird schliesslich wieder negativ. Im verlauf der Zeit führt dies zu einem Verschwinden der Diskrepanz zwischen natürlichen Preisen und effektiven preisen. Damit ist man am Beginn des nächsten zyklus. 
Vom klassisch-monetaristischen standpunkt aus haben die Schwankungen des Preisniveaus im Konjunkturzyklus einen stabilisierenden Effekt. Sofern man voraussetzen kann, daß Schwankungen des Preisniveaus keine Ruckwirkung auf die Umlaufgeschwindigkeit des Geldes haben, ist bei gegebener Entwicklung der Geldmenge die Höhe des nominellen Sozialprodukts im Konjunkturverlauf unabhängig von der Höhe des Preisniveaus. Je stärker dann die zyklischen schwankungen des Preisniveaus sind, desto geringer sind die schwankungen des realen sozialprodukts. Stellt man sich auf diesen klassischen standpunkt, so wäre die wirkung des Lizenzsystems, das das Preisniveau zyklisch kaum schwanken låsst, schlecht, da nun das reale sozialprodukt umso stärker schwanken muisste. In wirklichkeit gibt es aber eine sehr erhebliche Rückwirkung der Preise auf die umlaufgeschwindigkeit. Es ist eines der wesentlichen Verdienste von keynes und seiner Theorie, uns die Augen für die kurzfristige Flexibilität der Umlaufgeschwindigkeit des Geldes geöffnet zu haben. Es liegt im wesen der Liquidität - dazu ist sieja da - kurzfristig schwanken zu können, um Diskrepanzen zwischen Einnahmen und Ausgaben aufzufangen, a.h. um in gewissen Grenzen die Ausgaben unabhängig von den Einnahmen gestalt en zu können. Eine Verminderung der umlaufgeschwindigkeit des Geldes ist damit kurzfristig primär Folge und nicht Ursache abnehmender Gůternachfrage, ebenso wie umgekehrt eine erhöhte Kaufbereitschaft zuerst einmal die Umlaufgeschwindigkei des Geldes erhöht, also auch bei gegebener Geldmenge in tatsåchlich höheren Kåufen ihren Niederschlag findet. Erst wenn dann die Iiquiditätsversorgung ein ganzes stick von ihrem Normalwert abweicht, beginnt sie sich bei kaufentscheidungen stärker bemerkbar zu machen, weswegen die Geldmengensteuerung, mittel- bis langfristig, naturlich einen entscheidenden Einfluss auf die Höhe des nominellen sozialprodukts hat.

Wenn wir nun die Kaufbereitschaft der Wirtschaftssubjekte nicht primär durch ihre liquiditätsversorgung erklären, wovon hängt sie dann ab? Wir können dieser Frage nicht im Detail nachgehen. 
Hier ist vor allem wichtig, festzuhalten, daß die Nachfrage durch die Preise und die Prelserwartung mitbestimmt wird. Auch hier könnte man vom orthodoxen standpunkt aus sagen: je höher die Preise, desto niedriger die Nachfrage, so daß die konjunkturellen Preisschwankungen doch einen stabilisierenden Effekt auf die Nachfxage haben sollten. Wir wissen abef, daß in einer von Inflation geprägten welt (auch in einer von Deflation geprägten, wie uns die historische Erfahrung lehrt) die preiserwartungen ganz wesentilch durch die beobachtete Preisentwicklung bestimnt werden. Wenn nun in der Hochkonjunktur die Preise steigen, so fuihrt dies zur Erwartung noch weiter steigender Preise, die es als gůnstig erscheinen lassen, Kåufe so frůh als mo̊glich durchzufuhren, was die Konjunktur weiter anheizt. Ungekehrt beobachtet man in Rezessionen bei abflauender Preissteigerungsrate, daß dieser nachfragestimulierende Effekt der Preiserwartungen ausbleibt. In unserer Welt, die - mit Recht, wie man sagen muss - von der Inflationspsychologie geprågt ist, verlieren die konjunkturell bedingten Preisschwankungen ihre stabilisierende wirkung. Sie wirken vielmehr destabilisierend. Von daher ist unser Vorschlag der Kettung des erlaubten Preisniveaus an die Geldmenge auch unmittelbar konjunkturell von ginstiger wirkung.

Es muß aber betont werden, daß dies nicht das hauptsachliche Motiv für den Vorschlag ist. Das hauptsåchliche Motiv ist das der indirekten wirkung auf die preisentwicklung über den Einfluß auf die Tarifpolitik und insbesondere auf die Geldpolitik. Die stabilisierung der preise in der Hochkonjunktur mittels dieses Vorschlages und bei entsprechender Geldpolitik wird eher zu stabilitåtsadåquaten Lohnabschlưssen führen und diese wiederum machen eine Fortsetzung der stabilitätsadåquaten vergleichsweise restriktiven Geldpolitik ohne Gefährdung der Vollbeschäftigung möglich. Kann vermittels dieses Vorschlages eine politik des stabilen Geldes politisch durchgehalten werden, so wird dies letztlich auch eine stabilisierende Wirkung auf die Konjunkturentwicklung haben. 